FIAN/TD/20-05

\title{
Geometric Formulation for Partially Massless Fields
}

\author{
E.D. SkVortsovin And M.A. VAsiliev2 \\ I.E.Tamm Department of Theoretical Physics, P.N.Lebedev Physical Institute, \\ Leninsky prospect 53, 119991, Moscow, Russia
}

\begin{abstract}
The manifestly gauge invariant formulation for free symmetric higher-spin partially massless fields in $(A) d S_{d}$ is given in terms of gauge connections and linearized curvatures that take values in the irreducible representations of $(o(d-1,2)) o(d, 1)$ described by two-row Young tableaux, in which the lengths of the first and second row are, respectively, associated with the spin and depth of partial masslessness.
\end{abstract}

PACS: 11.15.-q;11.10.Kk

Keywords: Higher-spin fields gauge theory; partially-massless fields; (anti)-de Sitter space;

ArXiv.org:hep-th/0601095

\footnotetext{
${ }^{1}$ skvortsov@lpi.ru

${ }^{2}$ vasiliev@lpi.ru
} 


\section{Contents}

1 Introduction 3

2 Metric Formalism for Free Higher-Spin Fields 4

2.1 Massive higher-spin fields . . . . . . . . . . . . . . 5

2.2 Massless higher-spin fields . . . . . . . . . . . . . . . 6

2.3 Partially massless higher-spin fields . . . . . . . . . . . . . 6

2.3.1 The approach of Deser and Waldron . . . . . . . . . 7

2.3.2 The approach of Zinoviev ............... 9

3 Massless Higher-Spin Fields in the Frame-Like Formalism

3.1 Lorentz covariant approach . . . . . . . . . . . . . . . 10

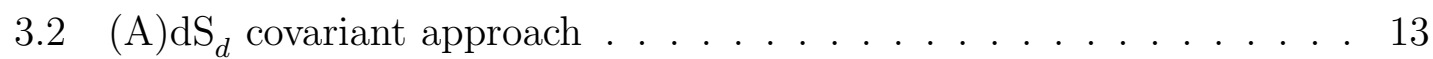

4 Partially Massless Higher-Spin Fields in the Frame-Like Formalism 17

4.1 Summary of results . . . . . . . . . . . . . . . . . 17

$4.2 \quad(\mathrm{~A}) \mathrm{dS}_{d}$ covariant action . . . . . . . . . . . . . . . 19

4.3 Lorentz covariant formulation . . . . . . . . . . . . . . . . 22

5 Examples $\quad 25$

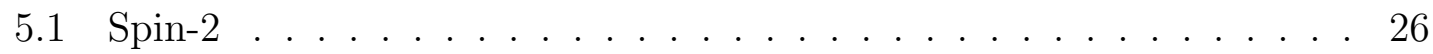

5.2 Spin-3 . . . . . . . . . . . . . . . . . . . . . . 28

6 Discussion $\quad 33$

A Appendix A: Notation

A.1 Index conventions . . . . . . . . . . . . . . . . . . 35

A.2 Condensed Notation . . . . . . . . . . . . . . . 36

B Appendix B: Young tableaux 36

C Appendix C: Coefficients 38 


\section{Introduction}

An interesting property of massive higher-spin (HS) fields in spaces with nonzero curvature like de Sitter $(d S)$ and anti-de Sitter $(A d S)$ is that for certain values of the parameter of mass, the HS field equations acquire gauge invariance. This phenomenon was originally discovered by Deser and Nepomechie in [1] and investigated further in [2]-[8]. The special values of the mass parameter are scaled in terms of the curvature of the background space. The first special value of the mass corresponds to the usual massless fields with the richest gauge symmetries. The other special values correspond to different fields called partially massless fields. A gauge transformation law of a partially massless field has less parameters but more derivatives compared to the massless fields. A number of degrees of freedom carried by a partially massless field is intermediate between the massless case (two for $s>0$ in $4 d$ ) and the massive case $(2 s+1$ in $4 d)$. In the flat space limit all special values of the mass shrink to zero so that one is left with the usual massless fields, i.e., partially massless theories do not exist in Minkowski space.

In $A d S_{d}$ space-time, the phenomenon of partial masslessness has clear interpretation in terms of representations of the $A d S_{d}$ algebra $o(d-1,2)$. Namely, a space of single-particle states of a given relativistic field with the energy bounded from below forms a lowest weight $o(d-1,2)$-module, $\left|E_{0}, s\right\rangle$. Its lowest weight is defined in terms of the lowest energy $E_{0}$ and spin $s$ associated with the weights of the maximal compact subalgebra $o(2) \oplus o(d-1) \subset o(d-1,2)$. For quantum-mechanically consistent fields, the modules $\left|E_{0}, s\right\rangle$ correspond to unitary representations of $o(d-1,2)$ that requires in particular $E_{0} \geq E_{0}(s)$ where $E_{0}(s)$ is some function of the spin $s$ found for the case of $d=4$ in [9] and for the general case in [10] (see also [11, 12, 13, 14, 15]). At $E_{0}=E_{0}(s)$ null states appear that form a submodule to be factored out. These signal a gauge symmetry of the system. The corresponding fields are the usual massless fields. Modules with lower energies $E<E_{0}(s)$ correspond to nonunitary (ghost) massive fields. At certain values $E_{i}(s)$ of $E$ the corresponding nonunitary module may contain a submodule that again signals a gauge symmetry in the fieldtheoretical description. Such modules correspond to the partially massless fields. Unfortunately, the lack of unitarity [8] makes their physical applications problematic in the $A d S$ case.

In the $d S_{d}$ case with the symmetry algebra $o(d, 1)$ the analysis is different because it admits no unitary lowest weight (i.e., bounded energy) representations at all. Relaxing the concept of bounded energy, it is possible to have unitary partially massless fields in that case [2, 4, 8].

In this paper we address the question what is a frame-like formulation of the 
symmetric partially massless fields, that generalizes the frame-like formulation of ordinary symmetric massless fields elaborated in [16, 17, 18, 19]. Such a reformulation operates in terms of a gauge field (connection), that takes values in some module of the $(A) d S_{d}$ algebra and makes gauge symmetries of the system manifest allowing to write down manifestly gauge invariant geometric actions. As shown in [19], a usual spin- $s$ massless field in $(A) d S_{d}$ is described by a 1 -form connection, which takes values in the finite-dimensional $o(d-1,2)$-module spanned by traceless tensors of $o(d-1,2)$ that have a symmetry of a length $s-1$ two-row rectangular

Young tableau \begin{tabular}{c|c}
\hline$s-1$ \\
\hline$s-1$
\end{tabular} . The main result of this paper is that a spin-s partially massless symmetric gauge fields is analogously described by a 1 -form connection, which is a $o(d-1,2)(o(d, 1))$-tensor that has the symmetry properties of a two-row Young tableaux with the first row of length $s-1$ and the second row of an arbitrary

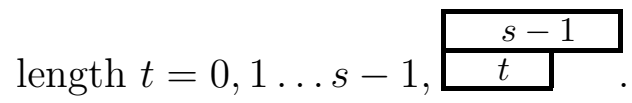

Since the gauge fields of the frame-like formalism are interpreted as connections of a non-Abelian HS algebra of a full HS system, the results of such a reformulation may be useful to rule out HS algebras incompatible with unitarity by requiring that physically relevant algebras considered as $o(d-1,2)$-modules under its adjoint action should not contain the modules associated with (nonunitary) partially massless fields. (Note that, as shown in [19], [20], gauge fields associated with true massless fields in $A d S_{d}$ carry $o(d-1,2)$ indices of various $o(d-1,2)$-modules described by Young tableaux, that have first two rows of equal lengths.) Although in this paper we consider integer spins, our approach admits a straightforward extension to fermions.

The layout of the rest of the paper is as follows. In Section 2 we recall the metriclike formulation of massive, massless and partially massless fields. The frame-like formalism for massless HS fields is recalled in Section 3. The main results of our approach are presented in Section 4 and then illustrated in Section 5 by the spin2 and spin-3 examples. Possible further developments are discussed in Section 6 . Notation, Young tableau convention and explicit expressions for some coefficients are collected in appendices.

\section{Metric Formalism for Free Higher-Spin Fields}

In this section we summarize known results on the description of massive, massless and partially massless HS fields, which are relevant to our analysis. 


\subsection{Massive higher-spin fields}

A massive spin- $s$ totally symmetric field in the flat Minkowski space can be described by a rank- $s$ symmetric tensor field $\phi_{\underline{m}_{1} \ldots \underline{m}_{s}}^{\{s\}}(x)$ that satisfies the equations [21] 1

$$
\begin{aligned}
& \left(\square+m^{2}\right) \phi^{\{s\}}=0, \\
& \partial \cdot \phi^{\{s\}}=\operatorname{divergence}\left(\phi^{\{s\}}\right)=0, \quad s \geq 1, \\
& \phi^{\{s\}^{\prime}}=\operatorname{trace}\left(\phi^{\{s\}}\right)=0, \quad s \geq 2,
\end{aligned}
$$

which form a complete set of local Lorentz-invariant conditions that can be imposed on $\phi^{\{s\}}$. The first equation of (2.1) is the mass-shell condition. The third condition, that the field $\phi^{\{s\}}$ is traceless, is an algebraic constraint. As pointed out by Fierz and Pauli in the pioneering paper [22], the second condition, which is a differential equation, can be derived for $s>1$ from a Lagrangian without introducing new degrees of freedom by adding auxiliary fields that are expressed in terms of (derivatives of) the dynamical fields by virtue of equations of motion. For totally symmetric massive fields of integer spins, the lagrangian formulation with a minimal set of auxiliary fields was worked out by Singh and Hagen in [23]. For a spin $s$ they introduced the set of auxiliary fields that consists of symmetric traceless tensors of ranks $s-2, s-3, \ldots 0$.

For example, to describe a spin-2 field, one auxiliary scalar field is needed. It can be identified with the trace of a traceful rank- 2 field $\phi_{\underline{m n}}$ satisfying the Fierz-Pauli equation

$$
G_{2}=\left(\square+m^{2}\right)\left(\phi_{\underline{m n}}-\eta_{\underline{m n}} \phi^{\prime}\right)+\partial_{\underline{m}} \partial_{\underline{n}} \phi^{\prime}+\eta_{\underline{m n}} \partial^{\underline{l}} \partial \underline{p} \phi_{\underline{l \underline{p}}}-2 \partial_{(\underline{m}} \partial^{\underline{k}} \phi_{\underline{k n})}=0 .
$$

One obtains

$$
\partial \cdot G_{2}=m^{2}\left(\partial \cdot \phi-\partial \phi^{\prime}\right)
$$

and

$$
\partial^{2} \cdot G_{2}-\frac{m^{2}}{d-2} G_{2}{ }^{\prime}=m^{4} \frac{d-1}{d-2} \phi^{\prime} .
$$

From equation (2.4) it follows that, if $m^{2} \neq 0, \phi$ is traceless (i.e., the auxiliary field is zero on-shell). Then from (2.3) it follows that $\partial \cdot \phi=0$.

If $m^{2}=0$, equation (2.3) expresses Bianchi identities which manifest the spin-2 gauge symmetry. Let us note that it is important that the higher-derivative part of the massive equations is the same as for massless fields just to make it possible to express the auxiliary fields in terms of the dynamical fields by virtue of algebraic constraints, that would not be possible for a different choice of the coefficients in

\footnotetext{
${ }^{1}$ Our notations and conventions are explained in Appendix A.
} 
front of the second derivative terms in (2.2). This means that, for a different choice of the coefficients, the corresponding equations describe a dynamical system with the propagating trace field, that possesses more degrees of freedom. A similar argument will play crucial role in the subsequent analysis of a proper action for a partially massless field.

Note that equivalent dynamical systems can be formulated with the aid of different sets of auxiliary fields. Particularly useful approaches to massive fields with different sets of auxiliary fields include the gauge invariant formulation of [5], [24] and the BRST approach of [25, 26].

\subsection{Massless higher-spin fields}

The free action for a symmetric massless field of an arbitrary integer spin in the flat Minkowski space was obtained by Fronsdal [27]. A symmetric spin- $s$ massless field is described by a totally symmetric double traceless tensor field $\phi^{\{s\}} \equiv \phi_{\underline{m}_{1} \ldots \underline{m}_{s}}(x)$, $\phi^{\{s\}^{\prime \prime}}=0$. The equations of motion

$$
\mathcal{F}^{s}=\square \phi^{\{s\}}-\partial \partial \cdot \phi^{\{s\}}+\partial^{2} \phi^{\{s\}^{\prime}}=0
$$

are invariant under gauge transformations

$$
\delta \phi^{\{s\}}=\partial \xi^{\{s-1\}}
$$

with a gauge parameter $\xi^{\{s-1\}}(x)$ being a symmetric rank- $(s-1)$ traceless tensor field. The gauge invariant Lagrangian is

$$
\mathcal{L}^{s}=\frac{1}{2} \phi^{\{s\}} \cdot \mathcal{F}^{s}-\frac{1}{8} s(s-1) \phi^{\{s\}^{\prime}} \cdot \mathcal{F}^{s^{\prime}}
$$

Note that alternative metric-like formulations of symmetric massless HS fields were also developed in [26, 28, 29].

\subsection{Partially massless higher-spin fields}

The $(\mathrm{A}) \mathrm{dS}_{d}$ space is described by a metric $g_{\underline{m n}}$ satisfying the equation

$$
R_{\underline{m n}, \underline{p q}}=-\frac{2 \Lambda}{(d-1)(d-2)}\left(g_{\underline{p} \underline{m}} g_{\underline{n} \underline{q}}-g_{\underline{p} \underline{\underline{n}}} g_{\underline{m} \underline{q}}\right) \text {, }
$$

where $R_{\underline{m n}, \underline{p q}}$ is the Riemann tensor and the normalization of the cosmological constant is chosen as in [4, 5]. $\Lambda$ is (negative)positive for (anti-)de Sitter space. Later on we use the notation $\lambda^{2}=-\frac{2 \Lambda}{(d-1)(d-2)}$, where $\left|\lambda^{-1}\right|$ is the $(\mathrm{A}) \mathrm{d} \mathrm{S}_{d}$ radius. 


\subsubsection{The approach of Deser and Waldron}

In this subsection, we consider the case of $d=4$ following to [4] (modulo the adjustment of the coefficients to the mostly minus signature conventions used in this paper). To illustrate the effect of partial masslessness let us start with the simplest example of spin two.

The deformation of the massive field equation (2.2) to $(\mathrm{A}) \mathrm{dS}_{d}$ is achieved by the addition of some mass-like terms proportional to $\Lambda$ along with the replacement of the flat derivatives by the covariant ones $D$ associated with $g_{\underline{m n}}$.

The $(\mathrm{A}) \mathrm{dS}_{d}$ deformation of the equation (2.2) is

$$
G_{2}=\left(\Delta^{(2)}+m^{2}-\Lambda\right)\left(\phi-g \phi^{\prime}\right)-\Lambda \phi+D D \phi^{\prime}-D D \cdot \phi+g D \cdot D \cdot \phi=0,
$$

where we use the notation of Deser and Waldron [4]

$$
\Delta^{(2)} \phi=\square \phi+\frac{8 \Lambda}{3}\left(\phi-\frac{1}{4} g \phi^{\prime}\right) .
$$

The equations (2.3) and (2.4) modify to

$$
\begin{gathered}
D \cdot G_{2}=m^{2}\left(D \cdot \phi-D \phi^{\prime}\right), \\
D^{2} \cdot G_{2}-\frac{1}{2} m^{2} G_{2}{ }^{\prime}=\frac{1}{2} m^{2}\left(3 m^{2}-2 \Lambda\right) \phi^{\prime} .
\end{gathered}
$$

One observes that, for the critical value $m^{2}=\frac{2}{3} \Lambda, \phi^{\prime}$ cannot be algebraically expressed in terms of the other field and the equation (2.12) becomes Bianchi identity signaling the appearance of a gauge invariance which is

$$
\delta \phi_{\underline{m n}}=\left(D_{(\underline{m}} D_{\underline{n})}-\frac{\Lambda}{3} g_{\underline{m n}}\right) \xi
$$

with a scalar gauge parameter $\xi$. This invariance kills the helicity zero degree of freedom leaving spin polarizations $\pm 1, \pm 2$. Note that although $\phi^{\prime}$ cannot be expressed in terms of the other field, this does not imply the appearance of extra degrees of freedom because it is pure gauge.

At $m=0,(2.11)$ is the Bianchi identity of the usual spin-2 massless theory in (A) $\mathrm{dS}_{d}$.

A spin-3 massive field requires a scalar auxiliary field $\chi$ and an auxiliary vector, which combines with a rank-3 traceless field into a rank-3 traceful tensor $\phi_{\underline{m n k}}$. The equations of motion of the massive spin-3 field $\phi_{\underline{m n k}}$ are [4]

$$
\begin{aligned}
G_{3}= & \left(\Delta^{(3)}+m^{2}-\frac{16 \Lambda}{3}\right) \phi-D D \cdot \phi+D D \phi^{\prime}- \\
& \quad-g\left(\left(\Delta^{(1)}+m^{2}-\frac{11 \Lambda}{3}\right) \phi^{\prime}-D \cdot D \cdot \phi+\frac{1}{2} D D \cdot \phi^{\prime}\right)-\frac{m}{4} g D \chi=0,
\end{aligned}
$$




$$
G_{0}=\frac{3}{2}\left(\Delta^{(0)}+4 m^{2}-8 \Lambda\right) \chi-m D \cdot \phi^{\prime}=0
$$

with

$$
\Delta^{3} \phi=\square \phi+5 \Lambda \phi-\frac{2 \Lambda}{3} g \phi^{\prime}, \quad \Delta^{(1)} \phi^{\prime}=\square \phi^{\prime}+\Lambda \phi^{\prime}, \quad \Delta^{(0)} \chi=\square \chi .
$$

The following identities hold

$$
\begin{gathered}
\Pi\left[D \cdot G_{3}\right]=\frac{1}{2} m \boldsymbol{\Pi}\left[\left(-D D \chi-m D \phi^{\prime}+m D \cdot \phi\right)\right], \\
D^{2} \cdot G_{3}+\frac{m}{4} D G_{0}-\frac{m^{2}}{4} G_{3}{ }^{\prime}=\frac{5}{8} m\left(3 m^{2}-4 \Lambda\right)\left(D \chi-\frac{2}{3} m \phi^{\prime}\right), \\
D^{3} \cdot G_{3}-\frac{5}{12} m\left(3 m^{2}-4 \Lambda\right) G_{0}=-\frac{5}{2} m\left(3 m^{2}-4 \Lambda\right)\left(m^{2}-2 \Lambda\right) \chi,
\end{gathered}
$$

where $\boldsymbol{\Pi}[\ldots]$ is the projector to the traceless part.

At $m=0$, (2.17) becomes the Bianchi identity associated with the gauge invariance with a rank-2 traceless parameter $\xi^{\{2\}}$

$$
\delta \phi=D \xi^{\{2\}}, \quad \delta \chi=0
$$

In this case, the system decomposes into two independent subsystems: massless spin three with two spin projections \pm 3 is described by the Fronsdal field $\phi$ and massive spin zero is described by $\chi$.

At $m^{2}=\frac{4}{3} \Lambda$, (2.18) becomes the Bianchi identity associated with the gauge invariance with the rank-1 gauge parameter $\xi^{\{1\}}$, which has the form

$$
\delta \phi=\left(D^{2}-\frac{\Lambda}{3} g\right) \xi^{\{1\}}, \quad \delta \chi=-\frac{2}{3} \sqrt{\frac{\Lambda}{3}} D \cdot \xi^{\{1\}} .
$$

This model describes a field with spin projections $\pm 2, \pm 3$.

At $m^{2}=2 \Lambda$, (2.19) becomes the Bianchi identity that manifests the gauge invariance with the scalar parameter $\xi^{\{0\}}$

$$
\delta \phi=\left(D^{3}-\frac{\Lambda}{2} g D\right) \xi^{\{0\}}, \quad \delta \chi=-\frac{2}{3} \sqrt{\frac{\Lambda}{3}}\left(D \cdot D-\frac{10 \Lambda}{3}\right) \xi^{\{0\}},
$$

leaving out the helicity zero degree of freedom. At non-critical $m^{2}$ the scalar $\chi$ and vector $\phi^{\prime}$ are auxiliary. They are zero on shell and $D \cdot \phi=0$ as a consequence of the constraints (2.17), (2.18) and (2.19). A generic non-critical system describes a massive spin-3 field with spin projections $(0, \pm 1, \pm 2, \pm 3$ ) (may be ghost and/or tachionic for negative $m^{2}$ ).

For general spin $s$, the full set of fields contains a rank- $s$ double traceless field along with the traceless fields of ranks from 0 to $s-3$. As argued in [4], there exist $s$ critical masses $m_{t}^{2}(t=0,1, \ldots, s-1)$ and the following properties are true for $m^{2}=m_{t}^{2}$ : 
- The equations of motion are invariant under the gauge transformation

$$
\delta \phi^{\{s\}}=\underbrace{D \ldots D}_{s-t} \xi^{\{t\}}+\ldots
$$

with $s-t$ derivatives and a rank- $t$ traceless gauge parameter $\xi^{\{t\}}$.

- A rank- $t$ traceless combination of the equations of motion becomes a Bianchi identity.

- The physical spin projections are $\pm(t+1), \ldots, \pm s$ plus decoupled lower spin massive fields.

\subsubsection{The approach of Zinoviev}

A useful approach to the description of massless, massive and partially massless fields in $(A) d S_{d}$ was proposed by Zinoviev in [5]. The Zinoviev's lagrangian is the sum of the covariantized Fronsdal's lagrangians $\mathcal{L}_{\text {cov }}^{s}$ (2.7) (with flat derivatives replaced by the covariant ones) for the set of spin- $k$ massless fields $\phi^{\{k\}}$ with $k=0 \ldots s$ plus some lower-derivative terms, i.e.,

$$
\mathcal{L}^{s}=\sum_{k=0}^{k=s} \mathcal{L}_{\text {cov }}^{k}+\triangle \mathcal{L}^{s}
$$

where

$$
\begin{gathered}
\triangle \mathcal{L}^{s}=\sum_{k=0}^{k=s}\left(a_{k} \phi^{\{k-1\}}\left(D \cdot \phi^{\{k\}}\right)+b_{k} \phi^{\{k\}^{\prime}}\left(D \cdot \phi^{\{k-1\}}\right)+c_{k}\left(D \cdot \phi^{\{k\}^{\prime}}\right) \phi^{\{k-1\}^{\prime}}+\right. \\
\left.+d_{k}\left(\phi^{\{k\}}\right)^{2}+e_{k}\left(\phi^{\{k\}^{\prime}}\right)^{2}+f_{k} \phi^{\{k\}^{\prime}} \phi^{\{k-2\}}\right) .
\end{gathered}
$$

The coefficients in $\triangle \mathcal{L}^{s}$ are fixed by the condition that $\mathcal{L}^{s}$ is invariant under some gauge transformations of the form

$$
\delta \phi^{\{k\}}=\frac{1}{k} D \xi^{\{k-1\}}+\alpha_{k} \xi^{\{k\}}+\beta_{k} g \xi^{\{k-2\}}
$$

with rank- $k$ traceless gauge parameters $\xi^{\{k\}}(k=0,1, \ldots, s-1)$. The gauge invariance condition expresses all the coefficients in terms of one free mass parameter $\mathrm{m}^{2}$.

For generic $m^{2}$, the lagrangian (2.24) describes a spin- $s$ massive field. In that case all gauge symmetries are Stueckelberg (i.e., algebraic for some of the fields). It is easy to see that the gauge fixing of all gauge symmetries reproduces the set of fields of Singh and Hagen [23], i.e., the Singh-Hagen theory deformed to $(A) d S_{d}$ is a gauge-fixed version of the theory of Zinoviev. In this approach it is still unclear, 
however, what is the fundamental principle that underlies the gauge symmetry of the model, thus fixing the coefficients in the action (2.24).

At the critical values of the mass [5]

$$
m_{t}^{2}=\frac{2 \Lambda}{(d-1)(d-2)}(s-t-1)(d+s+t-4), \quad t=0,1, \ldots, s-1
$$

some of the coefficients in the gauge transformation law (2.26) and in $\triangle \mathcal{L}^{s}$ vanish so that the lagrangian (2.24) splits into two independent parts. One contains the fields $\phi^{\{k\}}$ with $k=t+1 \ldots s$ and another one contains those with $k=0 \ldots t$. The former lagrangian describes a partially massless theory, including the usual massless theory as a particular case of $t=s-1$, while the latter describes a spin- $t$ massive field. In the flat limit $\Lambda \rightarrow 0$ all critical masses tend to zero and all lower derivative terms in the lagrangian vanish so that $\mathcal{L}^{s}$ reduces to the sum of the flat space Fronsdal's lagrangians of massless fields $\phi^{0}, \ldots, \phi^{s}$ of all spins from 0 to $s$.

\section{Massless Higher-Spin Fields in the Frame-Like Formalism}

\subsection{Lorentz covariant approach}

The Minkowski or $(\mathrm{A}) \mathrm{dS}_{d}$ geometry can be described by the frame field (vielbein) $e^{a}=d x \underline{\underline{m}} e_{\underline{m}}{ }^{a}$ and Lorentz spin-connection 1-form $\omega^{a, b}=d x \underline{\underline{m}} \omega_{\underline{m}}^{a, b}\left(\omega^{a, b}=-\omega^{b, a}\right)$, which obey the equations

$$
\begin{gathered}
T^{a} \equiv d e^{a}+\omega^{a,}{ }_{b} \wedge e^{b}=0, \\
R^{a, b} \equiv d \omega^{a, b}+\omega^{a,}{ }_{c} \wedge \omega^{c, b}-\lambda^{2} e^{a} \wedge e^{b}=0,
\end{gathered}
$$

where $d=d x \frac{\underline{m}}{\partial x \underline{\underline{m}}}$ is the de Rham operator, $-\lambda^{2}$ is the cosmological constant and the exterior product symbol $\wedge$ will be systematically omitted in what follows. The equation (3.1) is the zero-torsion constraint that expresses the Lorentz connection $\omega_{\underline{m}}^{a, b}$ via vielbein $e_{\underline{m}}^{a}$, provided that the vielbein is nondegenerate, which is required. The equation (3.2) then describes the $A d S_{d}$ space with the symmetry algebra $o(d-$ $1,2)$ if $\lambda^{2}>0, d S_{d}$ space with the symmetry algebra $o(d, 1)$ if $\lambda^{2}<0$ and flat Minkowski space if $\lambda^{2}=0$. The latter corresponds to the contraction of either $o(d-1,2)$ or $o(d, 1)$ to $i s o(d-1,1)$ and can be described by $\omega_{\underline{m}}^{a, b}=0$ and $e_{\underline{m}}{ }^{a}=\delta_{\underline{m}}{ }^{a}$ in Cartesian coordinates. Dynamical gravity is described by the vielbein and Lorentz connection which satisfy the zero-torsion condition (3.1) and the equations (3.2) relaxed to the Einstein equations. 
The Lorentz covariant derivative $D$ acts as usual 2

$$
\begin{aligned}
D C^{a(n), b(m), \ldots} & =d C^{a(n), b(m), \ldots}+n \omega^{a,}{ }_{c} C^{c a(n-1), b(m), \ldots}+ \\
& +m \omega^{b}{ }_{c} C^{a(n), c b(m-1), \ldots}+\ldots
\end{aligned}
$$

where $C^{a(n), b(m), \ldots,}(x)$ is an arbitrary Lorentz tensor field. As a consequence of (3.2), one obtains

$$
D^{2} C^{a(n), b(m), \ldots}=\lambda^{2}\left(n e^{a} e_{c} C^{c a(n-1), b(m), \ldots}+m e^{b} e_{c} C^{a(n), c b(m-1), \ldots}+\ldots\right) .
$$

Let us now recall how this approach extends to massless HS fields in flat and (A) $d S$ spaces. The generalization to HS massless fields in the Minkowski space [16] starts with the gauge fields $e_{\underline{m}}{ }^{a(s-1)}$ and $\omega_{\underline{m}}{ }^{a(s-1), b}$ with the tangent Lorentz indices possessing the symmetry properties of traceless Young tableaux of the form

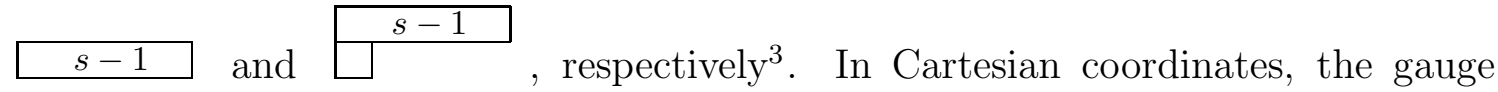
transformations are

$$
\begin{aligned}
& \delta \underline{\underline{m}}^{a(s-1)}=\partial_{\underline{m}} \xi^{a(s-1)}+\xi^{a(s-1),}, \underline{m} \\
& \delta \omega_{\underline{m}}{ }^{a(s-1), b}=\partial_{\underline{m}} \xi^{a(s-1), b}+\xi^{a(s-1), b} \underline{m},
\end{aligned}
$$

where the traceless gauge parameters $\xi^{a(s-1)}, \xi^{a(s-1), b}$ and $\xi^{a(s-1), b b}$ have the symmetry properties of Young tableaux $s-1, \square^{s-1}$ and $\square^{s-1}$, respectively.

In these terms the gauge invariant action can be written in the following simple form

$$
S=\int_{M^{d}} d x^{a_{1}} \ldots d x^{a_{d-3}} \varepsilon_{a_{1} \ldots a_{d-3} p q r}\left(d e^{n_{1} \ldots n_{s-2} p}+\frac{1}{2} d x^{m} \omega^{n_{1} \ldots n_{s-2} p,}{ }_{m}\right) \omega_{n_{1} \ldots n_{s-2}}^{q, r} .
$$

It is easy to check its gauge invariance, although it is not manifest.

Further analysis [17, 18] shows that, both in Minkowski and in $(\mathrm{A}) \mathrm{dS}_{d}$ cases, HS fields are conveniently described by a set of 1 -forms $\omega_{\underline{m}}{ }^{a(s-1), b(t)}, t=0,1, \ldots, s-$ 1 , possessing the symmetry properties of the two-row traceless Young tableaux 4 $s-1$ gauge fields for higher gauge symmetries the simplest of which is that with the gauge

\footnotetext{
${ }^{2}$ As explained in more detail in Appendix A, upper or lower indices denoted by the same letter are automatically symmetrized.

${ }^{3}$ I.e. the 1 -form $\omega^{a(s-1), b}$ is symmetric in the indices $a$, traceless in the indices $a$ and $b$, and the symmetrization over all indices gives zero: $\omega^{a(s-1), a} \equiv 0$. See also Appendix B.

${ }^{4}$ I.e. $\quad \omega^{a(s-1), b(t)}$ is symmetric in indices $a$ and $b$ separately, traceless in all indices and $\omega^{a(s-1), a b(t-1)} \equiv 0$.
} 
parameter $\xi^{a(s-1), b b}$ in the gauge transformations (3.5). This leads to the gauge field $\omega^{a(s-1), b b}$ which in its gauge transformation contains a new gauge parameter thus leading to the next extra field and so on.

The gauge transformation law for $e_{\underline{m}}^{a(s-1)} \equiv \omega_{\underline{m}}{ }^{a(s-1)}$ is

$$
\delta \omega_{\underline{m}}^{a(s-1)}=D_{\underline{m}} \xi^{a(s-1)}+e_{\underline{m} c} \xi^{a(s-1), c}
$$

where $D_{\underline{m}}$ is the Lorentz covariant derivative defined with respect to the background spin connection $\omega^{a, b}$ and vielbein $e^{a}$ that describe either Minkowski or (anti-)de Sitter geometry. Raising a form index by the vielbein $\omega^{a(s-1) \mid b}=\omega_{\underline{m}}{ }^{a(s-1)} e^{\underline{m} \mid b}$ one observes that this field decomposes into the following irreps of $o(d-1,1)$

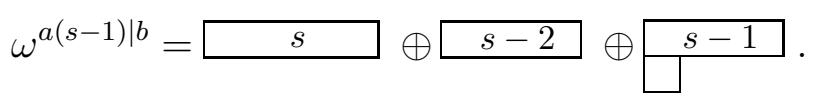

The first two components combine into the Fronsdal field: a rank-s double traceless tensor decomposes into traceless tensors of ranks $s$ and $(s-2)$. The last component is Stueckelberg. It is gauged away by the traceless gauge parameter $\xi^{a(s-1), c}$ that has the same symmetry type. As a result only the gauge parameter $\xi^{a(s-1)}$ is left and (3.7) gives rise to the Fronsdal gauge transformation law (2.6) .

With some rescaling of fields by $\lambda$ the gauge transformations of the other connections have the form

$$
\delta{\omega_{\underline{m}}}^{a(s-1), b(t)}=D_{\underline{m}} \xi^{a(s-1), b(t)}+\lambda e_{\underline{m} c} \xi^{a(s-1), b(t) c}+\lambda a_{s, t}(d) \mathcal{P}\left(\xi^{a(s-1), b(t-1)} e_{\underline{m}}^{b}\right),
$$

where $a_{s, t}(d)$ are some coefficients and the projector $\mathcal{P}$ restores the tracelessness and the proper Young symmetry. All the parameters $\xi^{a(s-1), b(t)}$ with $t>0$ are Stueckelberg, eliminating some components of the connections.

The field-strengths, which have analogous form 5

$$
R_{\{2\}}^{a(s-1), b(t)}=D \omega_{\{1\}}^{a(s-1), b(t)}+\lambda e_{c} \omega_{\{1\}}^{a(s-1), b(t) c}+\lambda a_{s, t}(d) \mathcal{P}\left(\omega_{\{1\}}^{a(s-1), b(t-1)} e^{b}\right),
$$

are demanded to be gauge invariant,

$$
\delta R_{\{2\}}^{a(s-1), b(t)}=0
$$

which condition fixes the coefficients $a_{s, t}(d)$.

The frame-like HS field $\omega_{\underline{m}}{ }^{a(s-1)}$ is called dynamical. The rest of the fields $\omega_{\underline{m}^{m}}{ }^{a(s-1), b(t)}, t=1,2, \ldots,(s-1)$ are expressed in terms of derivatives of the dynamical ones by virtue of certain constraints [18] which set to zero some components of

\footnotetext{
${ }^{5} \mathrm{~A}$ label in lower braces indicates the rank of a form; see Appendix A.
} 
the HS curvatures $R_{\{2\}}^{a(s-1), b(t)}$. A length $t$ of the second row of the Young tableau associated with a particular connection equals to the highest order of derivatives of the dynamical field in the resulting expressions for the higher connections

$$
\omega^{a(s-1), b(t)} \sim\left(\frac{\partial}{\lambda \partial x}\right)^{t} \omega^{a(s-1)}+\ldots .
$$

For example, in the spin-2 case, the field $\omega^{a, b}$ is the Lorentz connection. It is expressed in terms of the first derivatives of the dynamical field, the vielbein $e^{a}$. The field $\omega^{a(s-1), b}$ is analogous to the Lorentz connection and is called auxiliary. The condition that the free action contains at most two space-time derivatives of the dynamical field is equivalent to the extra field decoupling condition that requires the variation of the free action with respect to the extra fields to vanish identically

$$
\frac{\delta S}{\delta \omega^{a(s-1), b(t)}} \equiv 0, \quad t \geq 2
$$

A general $P$-even, gauge invariant action in $(\mathrm{A}) \mathrm{dS}_{d}$ has the form [17, 18]

$$
S=\frac{1}{2 \lambda} \sum_{k=0}^{k=s-2} a_{q} \int_{M^{d}} \epsilon_{c_{1} \ldots c_{d}} e^{c_{5}} \ldots e^{c_{d}} R_{\{2\}}{ }^{c_{1} a(s-q-2), c_{2} b(s-2)} R_{\{2\}}{ }^{c_{3}}{ }_{a(s-q-2)}{ }^{, c_{4}}{ }_{b(s-2)} .(3 .
$$

The extra field decoupling condition fixes the coefficients $a_{q}$ modulo a spin-dependent normalization factor. The resulting action is manifestly gauge invariant by (3.11), containing just two derivatives of the dynamical field upon excluding the auxiliary field by virtue of its equation of motion. As such, it is equivalent to the Fronsdal action in $A d S_{d}$. (In fact, the free action for massless fields in $A d S_{d}$ for any $d$ was originally obtained in this form in [18]).

\section{$3.2 \quad(\mathrm{~A}) \mathrm{dS}_{d}$ covariant approach}

The equations (3.1) and (3.2) are equivalent to the zero-curvature condition for the 1-form connection $W_{0}^{A, B}$ of $o(d-1,2)$ or $o(d, 1)$

$$
d W_{0}^{A, B}+W_{0}^{A,}{ }_{C} W_{0}^{C, B}=0 .
$$

For definiteness, let us consider the $A d S_{d}$ case of $o(d-1,2)$. The Lorentz connection and frame 1-form are identified as

$$
W_{0}^{a, b}=\omega^{a, b}, \quad W_{0}^{a, \bullet}=\lambda e^{a} .
$$

- denotes the extra time value of the $o(d-1,2)$ vector index compared to the $o(d-1,1)$ vector index. (For more detail see Appendix A.) The dimensionful 
constant $\lambda$ to be identified with the inverse $(A) d S$ radius is introduced to make the vielbein dimensionless. The curvature $R=d W_{0}+W_{0}^{2}$ decomposes as $R=$ $T^{a} P_{a}+\frac{1}{2} R^{a, b} M_{a, b}$, where the $o(d-1,2)$ generators $P_{a}$ and $M_{a, b}$ are associated with (A) $\mathrm{dS}_{d}$ "translations" and Lorentz rotations, respectively. $T^{a}$ and $R^{a, b}$ are the torsion tensor (3.1) and the modified Riemann tensor (3.2), respectively. The equation (3.15) can be rewritten as the nilpotency condition $D_{0}{ }^{2}=0$ for $D_{0}=d+W_{0}$.

As shown by MacDowell and Mansouri [30], modulo the topological GaussBonnet term, the action 6

$$
S=\frac{1}{4 \kappa^{2} \lambda^{2}} \int_{M^{d}} R^{a_{1}, a_{2}} R^{a_{3}, a_{4}} e^{a_{5}} \ldots e^{a_{d}} \epsilon_{a_{1} a_{2} \ldots a_{d}}
$$

in $d=4$ describes Einstein gravity with the cosmological term. The case of arbitrary $d$ was discussed in [19], where it was shown in particular that this action still describes a massless spin two field at the linearized level although differs from the pure Einstein action with the cosmological term at the nonlinear level (for more detail see also [31]).

A covariant generalization of the MacDowell-Mansouri approach proposed by Stelle and West [32] is achieved by introducing a compensator field $V^{A}(x)$ that has a fixed norm

$$
V^{A} V_{A}= \pm 1
$$

and carries no physical degrees of freedom. A sign $(+)$ - corresponds to the $(A) d S$ case. The Lorentz algebra $o(d-1,1) \subset o(d-1,2)(o(d, 1))$ is identified with the stability subalgebra of the compensator. The vielbein and spin connection admit the covariant definition

$$
\begin{gathered}
\lambda E^{A}=D V^{A}=d V^{A}+W_{0}{ }^{A}{ }_{B} V^{B}, \\
W_{0 L}{ }^{A, B}=W_{0}{ }^{A, B}-\lambda\left(E^{A} V^{B}-E^{B} V^{A}\right) .
\end{gathered}
$$

As a consequence of (3.19) and (3.18) the vielbein is orthogonal to the compensator

$$
E^{A} V_{A}=0
$$

and the Lorentz covariant derivative of the compensator with respect to the spin connection (3.20) is zero

$$
D_{L} V^{A}=d V^{A}+W_{0 L}^{A,}{ }_{B} V^{B}=0 .
$$

\footnotetext{
${ }^{6}$ the coefficient $\lambda^{2}$ instead of $\lambda$ is due to a somewhat different dimension of fields describing background space in comparison with dynamical ones. $\kappa^{2}$ is the gravitational constant.
} 
A convenient gauge choice, referred to as standard gauge, which breaks local $o(d-1,2)$ $(o(d, 1))$ down to the local Lorentz algebra, is

$$
V^{A}=\delta_{\bullet}^{A}
$$

with the compensator vector pointing along the extra $(d+1)^{t h}$ direction. In the standard gauge, the nonzero components $E^{a}$ of $E^{A}$ are identified with the the vielbein $e^{a}$.

The idea of the approach to the description of HS gauge fields proposed in [18, 19] was to replace the $(\mathrm{A}) \mathrm{dS}_{d}$ isometry algebra $h=o(d-1,2)$ or $o(d, 1)$ by some its extension $g$ called HS symmetry algebra in such a way that the dynamics of massless HS fields is described in terms of the connection 1-forms of $g$ similarly to as gravity is described by the connections of $o(d, 1)(o(d-1,2))$. Note that $g$ turns out to be infinite-dimensional.

The free field approximation results from the following perturbative expansion. A zero-order vacuum (background) field $\omega_{0}$ takes values in $h \subset g$, i.e., it identifies with the 1 -form $W_{0}^{A, B}=-W_{0}^{B, A}$. It is required to satisfy the zero-curvature equation for $h$ thus describing (A) $\mathrm{dS}_{d}$ space-time (or Minkowski in the flat limit).

$\omega_{1}$ describes first-order fluctuations of the fields of all spins including gravity. The HS field strength is

$$
R=R_{0}+R_{1}+o\left(\omega_{1}\right)
$$

where

$$
\begin{aligned}
& R_{0}=d \omega_{0}+\left[\omega_{0}, \omega_{0}\right], \\
& R_{1}=d \omega_{1}+\left[\omega_{0}, \omega_{1}\right] .
\end{aligned}
$$

Here [,] is the Lie product of $g$. Since $h \subset g$, the zero-order part of the curvature of the full HS algebra $g$ is zero $R_{0}=0$. The first-order part is $R_{1}=D_{0} \omega_{1}$, where $D_{0}$ is the background covariant derivative built of the connection $\omega_{0}$. Since $D_{0}{ }^{2}=0, R_{1}$ is invariant under the linearized gauge transformations

$$
\delta \omega_{1}=D_{0} \xi
$$

where $\xi$ is an arbitrary gauge parameter that takes values in $g$. This is the linearized HS gauge transformation. Any action constructed in terms of $R_{1}$ is therefore automatically HS gauge invariant at the linearized level.

In the known cases, $g$ decomposes into an infinite direct sum of finite-dimensional $h$-modules under the adjoint action of $h \subset g$. Gauge fields $\omega_{1}^{s}$, corresponding to different irreducible submodules labeled by the integral weights $s$ of $h$, describe fields of different "spins" s. In other words, the connections $\omega_{1}^{s}$ take values in irreducible tensor $h$-modules. 
As an example, a spin- $s$ symmetric massless field in $(\mathrm{A}) \mathrm{dS}_{d}$ can be described [19] by the 1-form $W_{\underline{m}}^{A(s-1), B(s-1)}$ with tangent indices that possess the symmetry of

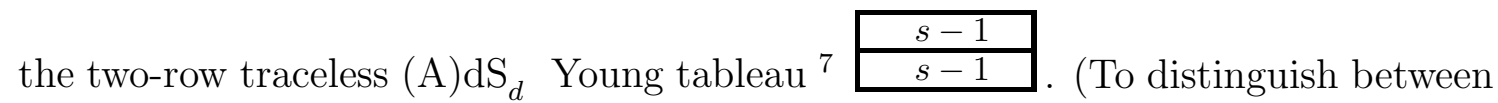
Young tableaux of $o(d-1,1)$ and $o(d-1,2)$ the latter are drown in bold.) The gauge transformation law is

$$
\delta W_{\{1\}}^{A(s-1), B(s-1)}=D_{0} \xi_{\{0\}}^{A(s-1), B(s-1)} .
$$

The field strength

$$
R_{\{2\}}^{A(s-1), B(s-1)}=D_{0} W_{\{1\}}^{A(s-1), B(s-1)}
$$

is gauge invariant by virtue of $D_{0}^{2}=0$.

To make contact with the analysis of [18] explained in Section 3.1] one observes

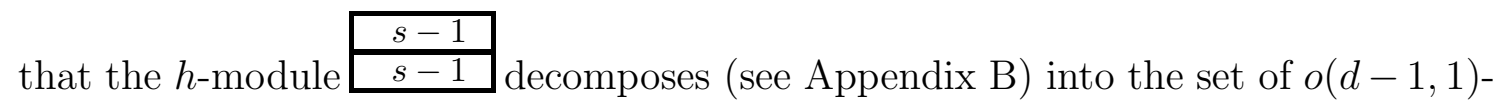
modules

$$
\begin{array}{|c|}
\hline s-1 \\
\hline k
\end{array}, k=0,1, \ldots,(s-1)
$$

i.e., $W_{\{1\}}^{A(s-1), B(s-1)} \longleftrightarrow \omega^{a(s-1), b(k)}, k=0,1, \ldots,(s-1)$. In particular, the vielbeinlike field and the Lorentz connection-like auxiliary field are

$\omega_{\underline{m}}{ }^{a(s-1)}=W_{\underline{m}}{ }^{a(s-1), C(s-1)} V_{C_{1}} \ldots V_{C_{s-1}}, \quad{\omega_{\underline{m}}}^{a(s-1), b}=W_{\underline{m}}{ }^{a(s-1), b C(s-1)} V_{C_{1}} \ldots V_{C_{s-2}}$.

In the $o(d-1,2)$ covariant approach, a general $P$-even HS gauge invariant action is

$$
\begin{aligned}
S^{s} & =\frac{1}{2 \lambda} \sum_{q=0}^{q=s-2} b_{q}^{s} \int_{M^{d}} \epsilon_{A_{1} \ldots A_{d+1}} V^{A_{5}} E^{A_{6}} \ldots E^{A_{d+1}} \times \\
& \times R_{\{2\}}{ }^{A_{1} B(s-2), A_{2} C(q) D(s-q-2)} R_{\{2\}}{ }^{A_{3}{ }_{B(s-2)},{ }^{A_{4} C(q)}{ }_{D(s-q-2)} V_{C(2 q)},}
\end{aligned}
$$

where $V_{C(p)}=\underbrace{V_{C} \ldots V_{C}}_{p}$. The coefficients $b_{q}^{s}$ are fixed [19] modulo an overall factor

$$
b_{q}^{s}=b^{s} \frac{(d-5+2 q) ! !(q+1)}{q !}
$$

by the condition that the variation with respect to the fields contracted with less than $s-2$ compensators is identically zero

$$
\Pi_{L}\left[V^{C(k)} \frac{\delta S}{\delta W^{A(s-1), B(s-k-1) C(k)}}\right] \equiv 0, \quad k<s-2,
$$

${ }^{7}$ I.e. the field $W_{\underline{m}}{ }^{A(s-1), B(s-1)}$ is symmetric in indices $A$ and $B$ separately, traceless and the symmetrization over any $s$ indices gives zero: $W_{\underline{m}}{ }^{A(s-1), A B(s-2)} \equiv 0$. 
where $\Pi_{L}$ is the projector to the $V^{A}$ - transversal part of a tensor. By virtue of (3.31), this condition is equivalent to the extra field decoupling condition (3.13). As a result, modulo total derivatives, the action depends only on the vielbein-like and Lorentz connection-like fields (3.31), being free of higher derivatives.

Now we are in a position to apply this approach to the description of partially massless fields. For more detail on the HS gauge theory we refer the reader to [31, 33.

\section{Partially Massless Higher-Spin Fields in the Frame-Like Formalism}

\subsection{Summary of results}

Our main result is that a free partially massless field of spin- $s$ and depth- $t$ can be described by a gauge field $W_{\{1\}}^{A(s-1), B(t)}$, which has the symmetry of two-row traceless

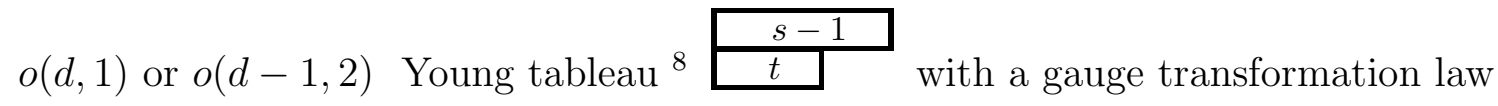

$$
\delta W_{\{1\}}^{A(s-1), B(t)}=D_{0} \xi_{\{0\}}^{A(s-1), B(t)}
$$

and a field strength

$$
R_{\{2\}}^{A(s-1), B(t)}=D_{0} W_{\{1\}}^{A(s-1), B(t)} .
$$

Since $D_{0}{ }^{2}=0$, the field strength is gauge invariant and satisfies Bianchi identities

$$
\delta R_{\{2\}}^{A(s-1), B(t)}=0, \quad D_{0} R_{\{2\}}^{A(s-1), B(t)}=0 .
$$

In the rest of this section we sketch the main results of our analysis leaving some details of their derivation to a later publication [34, where it will be shown in particular, that all gauge symmetry parameters $\xi_{\{0\}}^{A(s-1), B(t)}$ are Stueckelberg (i.e., analogous to the Lorentz symmetry parameters in the case of gravity) except for the parameter

$$
\xi^{b(t)}=\xi_{\{0\}}^{A(s-1), b(t)} V_{A_{1}} \ldots V_{A_{s-1}},
$$

the most $V$-tangential part of $\xi_{\{0\}}^{A(s-1), B(t)}$, which is traceless as a consequence of the tracelessness and the symmetry properties of $\xi_{\{0\}}^{A(s-1), B(t)}$ analogous to those of $W_{\{1\}}^{A(s-1), B(t)}$. Indeed, in the standard gauge $V^{A}=\delta_{\bullet}^{A}$

$$
\xi^{b(t)} \eta_{b b}=-\eta_{\bullet \bullet} \xi_{\{0\}}^{A(s-1), \bullet \bullet b(t-2)} V_{A_{1}} \ldots V_{A_{s-1}}=\xi_{\{0\}}^{\bullet \ldots \bullet \bullet \bullet b(t-2)} \equiv 0,
$$

\footnotetext{
${ }^{8}$ I.e. $W^{A(s-1), B(t)}$ is a traceless tensor symmetric in indices $A$ and $B$ separately, that satisfies the antisymmetry property $W^{A(s-1), A B(t-1)} \equiv 0$.
} 
since the symmetrization over any $s$ indices of $\xi_{\{0\}}^{A(s-1), B(t)}$ gives zero.

By dynamical fields we mean those which are neither pure gauge (i.e., Stueckelberg) nor auxiliary (i.e., to be expressed in terms of derivatives of the other fields by virtue of constraints). The dynamical fields in the system with $t<s-1$ are [34] traceless symmetric tensors $\phi^{a(s)}, \phi^{a(t)}$ and $\phi^{a(t-1)}$ (the latter field is absent for $t=0)$ defined as follows

$$
\phi^{a(s)}=\Pi\left[W^{a(s-1), C(t) \mid a} V_{C_{1}} \ldots V_{C_{t}}\right],
$$

where $\Pi[]$ is a projector to the traceless part,

$$
\phi^{a(t-1)}=W_{m}^{m a(t-1) C(s-t-1), C(t)} V_{C_{1}} \ldots V_{C_{s-1}}, \quad t>0
$$

and $\phi^{a(t)}$ is a linear combination of the form

$$
\phi^{a(t)}=\alpha W_{m}^{m a(t) C(s-t-2), C(t)} V_{C_{1}} \ldots V_{C_{s-2}}+\beta W_{m}^{a(t) C(s-t-1), C(t-1) m} V_{C_{1}} \ldots V_{C_{s-2}} .
$$

Note that the fields $\phi^{a(t)}$ and $\phi^{a(t-1)}$ are automatically traceless (cf. (4.5)).

The gauge transformation law for the field $\phi^{a(s)}$ is

$$
\delta \phi^{a(s)}=\Pi[\overbrace{D^{a} \ldots D^{a}}^{s-t} \xi^{a(t)}] .
$$

It coincides with the transformation law (2.23) for partially massless fields found by Deser and Waldron [4] and Zinoviev [5].

Gauge invariant combinations of derivatives of the dynamical fields that are nonzero on-shell we call generalized Weyl tensors. In practice, to find generalized Weyl tensors, one should set to zero as many components of the gauge invariant field strengths as possible by imposing constraints that express auxiliary fields in terms of derivatives of the dynamical fields (these generalize the zero-torsion condition in gravity). Other components of the gauge invariant field strengths are zero by virtue of the field equations (these generalize the Einstein equations in gravity). Some more vanish by virtue of Bianchi identities applied to the former two (e.g., the cyclic part of the Riemann tensor in gravity is zero by virtue of the Bianchi identity applied to the zero-torsion condition). What is left nonzero are the generalized Weyl tensors.

In the massless case with $t=s-1$, the generalized Weyl tensor is known [19] to be described by the length $s$ two-row rectangular $o(d-1,1)$ Young tableau. In the partially massless case, a generalized Weyl tensor identifies [34] with the traceless component of $R_{\{2\}}^{a(s-1), b(t)}$ (which is the $V^{A}$ orthogonal part of $R_{\{2\}}^{A(s-1), B(t)}$ ), which has the symmetry property of $\frac{s}{n .1}$. 
The case of $t=0$, which corresponds to the conformal generalization of the Maxwell theory discussed in [4], is special. The $t=0$ generalized Weyl tensors are the components of $R_{\underline{m n}}{ }^{a(s-1)}$ with the symmetry properties of the Young tableaux

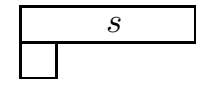
and $s-1$ for $s>1$.

The geometric formulation of the partially massless fields will be illustrated in Section 5 by the examples of $s=2, t=0$ with the gauge field $W_{\underline{m}}{ }^{A}$ and $s=3$, $t=1$ with the gauge field $W_{\underline{m}}^{A A, B}$. In the spin-2 example, the dynamical fields $\phi^{a(s)}, \phi^{a(t)}$ correspond to $\Pi\left[\bar{W}^{(a \mid a)}\right]$ and $W_{m}^{m \mid}$ while $\phi^{a(t-1)}$ is absent as $t=0$. In the spin-3 example $\phi^{a(s=3)}=\Pi\left[W^{(a a \mid a)}\right], \phi^{a(t=1)}=\alpha W_{m}^{a m, \bullet \mid}+\beta W^{[a \bullet, m] \mid}$ and $\phi^{a(t-1=0)}=W_{m}^{m \bullet \bullet \bullet}$.

\section{$4.2(\mathrm{~A}) \mathrm{dS}_{d}$ covariant action}

Free gauge invariant action functionals are easily constructed in terms of the gauge invariant linearized curvatures in the form

$$
\begin{aligned}
& S^{s, t}=\frac{1}{2 \lambda} \sum_{k, m} \tilde{b}_{k, m}^{s, t} \int_{M^{d}} \epsilon_{A_{1} A_{2} \ldots A_{d+1}} V^{A_{5}} E^{A_{6}} \ldots E^{A_{d+1}} \\
& R_{\{2\}}{ }^{A_{1} B(s-k-2) C(k), A_{2} D(t-m-1) C(m)} R_{\{2\}}{ }^{A_{3}} B(s-k-2){ }^{C(k), A_{4}}{ }^{(t-m-1)}{ }^{C(m)} V_{C(2 k+2 m)},
\end{aligned}
$$

where

$$
\tilde{b}_{k, m}^{s, t}=b_{k, m}^{s, t} \theta(m) \theta(k) \theta(t-m-1) \theta(s-m-k-2)
$$

with

$$
\begin{cases}\theta(n)=1 & n \geq 0, \\ \theta(n)=0 & n<0 .\end{cases}
$$

implies that the summation range is finite for a fixed spin.

The parameters $s$ and $t$, which determine a field type, are fixed. Different choices of the coefficients $b_{k, m}^{s, t}$ lead to different gauge invariant dynamical systems. To simplify formulae, the range of summation over the indices $k$ and $m$ in (4.10) is chosen so that the terms with $k>s-t-1$ are not independent, being expressible via linear combinations of terms with smaller $k$ because the symmetrization over any $s$ tangent indices of the HS curvatures gives zero.

Generally, different gauge connections that enter the curvatures in (4.10) are independent fields, which may carry their own degrees of freedom. Alternatively, all connections can be expressed in terms of (derivatives of) certain dynamical fields by virtue of imposing constraints that generalize the zero torsion condition in gravity and require some components of the field strengths to vanish. Plugging the resulting 
expressions back into the action gives rise to a gauge invariant higher derivative action, which may again describe a system with extra degrees of freedom. To avoid these unwanted degrees of freedom, the coefficients in the action (4.10) should be adjusted so that, in the flat limit, the action should amount to the sum of free massless actions for spins from $t+1$ to $s$ in agreement with the construction of Zinoviev discussed in Section 2.3.2. It turns out that for the models with $t>1$ this is achieved by imposing the extra field decoupling condition

$$
\Pi_{L}\left[V^{C(m+k)} \frac{\delta S^{s, t}}{\delta W^{A(s-k-1) C(k), B(t-m) C(m)}}\right] \equiv 0, \quad \begin{aligned}
& m=0,1, \ldots, t-2, \\
& k=0,1, \ldots, s-t-1
\end{aligned},
$$

which means that the variation is nonzero only for the components $W^{A(s-1), B C(t-1)} V_{C(t-1)}$ and generalizes (3.34) to partially massless fields.

Using

$$
\delta R_{\{2\}}^{A(s-1), B(t)}=D_{0} \delta W_{\{1\}}^{A(s-1), B(t)},
$$

the symmetry properties of the field strength, the Bianchi identities (4.3), the definition of the frame (3.19) along with its consequence $D_{0} E^{A}=0$ and the identities

$$
\begin{aligned}
& \epsilon_{A_{1} \ldots A_{d+1}}=V^{F}\left(V_{A_{1}} \epsilon_{F A_{2} \ldots A_{d+1}}+V_{A_{2}} \epsilon_{A_{1} F A_{3} \ldots A_{d+1}}+\ldots+V_{A_{d+1}} \epsilon_{A_{1} \ldots A_{d} F}\right), \\
& \epsilon_{A_{1} \ldots A_{5} H_{6} \ldots H_{d+1}} E^{F} E^{H_{6}} \ldots E^{H_{d+1}}= \\
& \frac{1}{d-3}\left(\sum_{k=1}^{k=5} \epsilon_{A_{1} \ldots \widehat{\mathbf{A}}_{k} \ldots A_{5} H_{5} \ldots H_{d+1}} \delta_{A_{k}}^{F}(-)^{k+1}\right) E^{H_{5}} \ldots E^{H_{d+1}},
\end{aligned}
$$

which manifest the fact that the antisymmetrization of $d+2$ indices, which take $d+1$ values, is zero, we obtain

$$
\begin{aligned}
& \delta S^{s, t}=\frac{1}{d-3} \sum_{k, m} \int_{M^{d}} \epsilon_{A_{1} A_{2} \ldots A_{d+1}} V^{A_{4}} E^{A_{5}} \ldots E^{A_{d+1}} V_{C(2 k+2 m+1)} \times \\
\times & \left\{\delta W^{A_{1} B(s-k-2) C(k), A_{2} D(t-m-1) C(m)} R_{B(s-k-2){ }^{A_{3}}{ }^{C(k),}{ }_{D(t-m-1)}^{C(m+1)} \times} \times\right. \\
\times & \left([d-3+2(k+m)] \tilde{b}_{k, m}^{s, t}-(m+1) \tilde{b}_{k, m+1}^{s, t}+\frac{(k+1)^{2}}{(s-k-2)} \tilde{b}_{k+1, m}^{s, t}\right)+ \\
+ & \delta W^{A_{1} B(s-k-2) C(k), A_{2} D(t-m-1) C(m)} R_{B(s-k-2)}^{C(k+1), A_{3}}{ }^{C(t-m-1)}{ }^{C(m)} \times \\
\times & \left(-[d-3+2(k+m)] \tilde{b}_{k, m}^{s, t}+\frac{(k+1)(s-k-m-1)}{(s-k-2)} \tilde{b}_{k+1, m}^{s, t}\right)+ \\
& +(\delta W \longleftrightarrow R)\} .
\end{aligned}
$$


The extra field decoupling condition (4.13) demands most of the terms in the variation (4.17) to vanish. This is achieved by virtue of imposing the following conditions on the coefficients

$$
\left\{\begin{array}{l}
-[d-3+2(k+m)] b_{k, m}^{s, t}+\frac{(k+1)(s-k-m-1)}{(s-k-2)} b_{k+1, m}^{s, t}=0 \\
{[d-3+2(k+m)] b_{k, m}^{s, t}-(m+1) b_{k, m+1}^{s, t}+\frac{(k+1)^{2}}{(s-k-2)} b_{k+1, m}^{s, t}=0} \\
t>1, k=0, \ldots, s-t-1, m=0, \ldots, t-2,
\end{array}\right.
$$

which fix $b_{k, m}^{s, t}$ up to an overall factor $b^{s, t}$

$$
b_{k, m}^{s, t}=b^{s, t} \frac{(s-k-m-1) !(d-5+2(k+m)) ! !}{k ! m !(s-k-2) !(s-m) !} .
$$

The remaining nonzero part of the variation of the action is

$$
\begin{aligned}
& \delta S^{s, t}=\frac{1}{d-3} \sum_{k} \int_{M^{d}} \epsilon_{A_{1} A_{2} \ldots A_{d+1}} V^{A_{4}} E^{A_{5}} \ldots E^{A_{d+1}} V_{C(2 k+2 t-1)} \times \\
\times & \left\{\delta W^{A_{1} B(s-k-2) C(k), A_{2} C(t-1)} R^{A_{3}}{ }_{B(s-k-2)}{ }^{C(k), C(t)}+(\delta W \longleftrightarrow R)\right\} \times \\
\times & \left([d-5+2(k+t)] \tilde{b}_{k, t-1}^{s, t}+\frac{(k+1)^{2}}{(s-k-2)} \tilde{b}_{k+1, t-1}^{s, t}\right) .
\end{aligned}
$$

As explained in Section 4.3, the coefficients (4.19) guarantee the consistency of the flat limit, which is in agreement with the construction of Zinoviev. For the massless case of $t=s-1$, the variation reduces to that of the action (3.32) [19].

The partially massless cases with $t=0$ and $t=1$ are special. In the case of $t=1$, the variation of the action is

$$
\begin{aligned}
& \delta S^{s, 1}=\frac{1}{d-3} \sum_{k} \int_{M^{d}} \epsilon_{A_{1} A_{2} \ldots A_{d+1}} V^{A_{4}} E^{A_{5}} \ldots E^{A_{d+1}} V_{C(2 k+1)}\{ \\
& \quad \delta W^{A_{1} B(s-k-2) C(k), A_{2}} R_{B(s-k-2)}^{A_{3}}{ }^{C(k), C}\left[(d-3+2 k) \tilde{b}_{k, 0}^{s, 1}+\frac{(k+1)^{2}}{(s-k-2)} \tilde{b}_{k+1,0}^{s, 1}\right]+ \\
& +\delta W^{A_{1} B(s-k-2) C(k), A_{2}} R_{B(s-k-2)}{ }^{C(k+1), A_{3}}\left[-(d-3+2 k) \tilde{b}_{k, 0}^{s, 1}+\frac{(k+1)(s-k-1)}{(s-k-2)} \tilde{b}_{k+1,0}^{s, 1}\right]+ \\
& +(\delta W \longleftrightarrow R)\} .
\end{aligned}
$$

The extra fields decoupling condition (4.13) is not applicable for this case and the coefficients are fixed directly from the requirement of consistency of the flat limit. As explained in Subsection 4.3, upon an appropriate rescaling, the first term in (4.21) tends in the flat limit to the sum of variations of massless actions for spins from $(t+1)$ to $s$, while the second term diverges. The correct flat limit therefore requires the second term to be zero that fixes $b_{k, 0}^{s, t}$ in the form

$$
b_{k, 0}^{s, 1}=b^{s, 1} \frac{(s-k-1)(d-5+2 k) ! !}{k ! s !},
$$


i.e., (4.19) is still true.

In the case of $t=0$, the action cannot be written at all in the form (4.10) because there is no room for the indices $A_{2}$ and $A_{4}$. For this case the action is constructed in terms of the generalized Weyl tensor $C^{a(s), b}$

$$
S^{s, 0}=\frac{1}{2} \int_{M^{d}} \epsilon_{c_{1} \ldots c_{d}} e^{c_{1}} \ldots e^{c_{d}}\left(C^{a(s), b} C_{a(s), b}-\frac{1}{2} C_{m}^{a(s-1) m,} C_{a(s-1) n,}{ }^{n}\right) .
$$

Note that, in $d=4$, this case corresponds to the conformal partially massless fields of Deser and Waldron [4] with 4d Maxwell electrodynamics as a particular case of $s=1$, where $C^{a, b}$ identifies with the Maxwell field strength. The form of the action (4.23) is in agreement with the well-known fact that the Maxwell lagrangian 4-form cannot be written as the exterior product of the Maxwell 2-form field strengths.

\subsection{Lorentz covariant formulation}

The practical analysis of the field content of the model under consideration is more illuminating in terms of the Lorentz irreducible components of the fields. An irrep

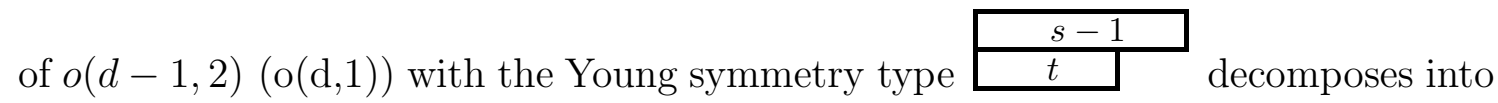
the following set of irreps of $o(d-1,1)$

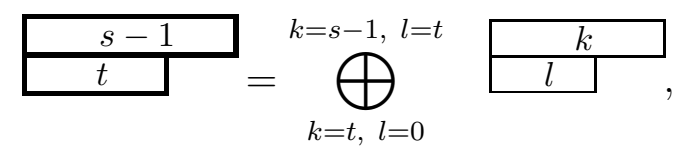

i.e., in terms of Lorentz tensors, the partially massless theory of spin- $s$ and depth- $t$ is described by 1 -forms $\omega_{\{1\}}^{a(k), b(l)} \equiv \omega_{\{1\}}^{\{k, l\}}$ with $k=t, \ldots, s-1, l=0,1, \ldots, t$.

The field strengths have the following structure

$$
\begin{aligned}
R_{\{2\}}^{\{k, l\}}=D_{0}\left(\omega_{\{1\}}^{\{k, l\}}\right) & =D\left(\omega_{\{1\}}^{\{k, l\}}\right)+\lambda \sigma_{-}^{1}\left(\omega_{\{1\}}^{\{k+1, l\}}\right)+\lambda \sigma_{-}^{2}\left(\omega_{\{1\}}^{\{k, l+1\}}\right)+ \\
& +\lambda \sigma_{+}^{1}\left(\omega_{\{1\}}^{\{k-1, l\}}\right)+\lambda \sigma_{+}^{2}\left(\omega_{\{1\}}^{\{k, l-1\}}\right) .
\end{aligned}
$$

The operators $\sigma_{ \pm}^{1,2}$ have the form

$$
\begin{aligned}
\sigma_{+}^{1}\left(C^{a(k), b(l)}\right) & =g(k, l)(k+1)\left(e^{a} C^{a(k), b(l)}-\frac{k}{2(d+2 k-2)} e_{m} C^{a(k-1) m, b(l)} \eta^{a a}-\right. \\
& -\frac{l}{d+k+l-3} e_{m} C^{a(k), m b(l-1)} \eta^{a b}+ \\
& \left.+\frac{k}{l(d+2 k-2)(d+k+l-3)} e_{m} C^{a(k-1) b, b(l-1) m} \eta^{a a}\right),
\end{aligned}
$$




$$
\begin{aligned}
& \sigma_{+}^{2}\left(C^{a(k), b(l)}\right)=G(k, l)(l+1)\left(e^{b} C^{a(k), b(l)}-\frac{k}{k-l} e^{a} C^{a(k-1) b, b(l)}+\right. \\
&-\frac{l}{d+2 l-4} e_{m} C^{a(k), b(l-1) m} \eta^{b b}+ \\
&-\frac{k(k-l-1)}{(k-l)(d+k+l-3)} e_{m} C^{a(k-1) m, b(l)} \eta^{a b}+ \\
&+\frac{l k(d+2 k-4)}{(k-l)(d+2 l-4)(d+k+l-3)} e_{m} C^{a(k-1) b, b(l-1) m} \eta^{a b}+ \\
&+\frac{k(k-1)}{(k-l)(d+k+l-3)} e_{m} C^{a(k-2) m b, b(l)} \eta^{a a}+ \\
&\left.-\frac{l k(k-1)}{(k-l)(d+k+l-3)(d+2 l-4)} e_{m} C^{a(k-2) b b, b(l-1) m} \eta^{a a}\right), \\
& \sigma_{-}^{1}\left(C^{a(k), b(l)}\right)=f(k, l)\left(e_{m} C^{a(k-1) m, b(l)}+\frac{l}{k-l+1} e_{m} C^{a(k-1) b, b(l-1) m}\right), \\
& \sigma_{-}^{2}\left(C^{a(k), b(l)}\right)=F(k, l)\left(e_{m} C^{a(k), b(l-1) m}\right),
\end{aligned}
$$

where $g(k, l), G(k, l), f(k, l)$ and $F(k, l)$ are some coefficients, which can be either derived from the covariant field strengths (4.2) or obtained directly from the condition of the gauge invariance of $R_{\{2\}}^{a(k), b(l)}$. Their explicit form is given in Appendix C. (For more detail see [34]).

A general $P$-even $o(d-1,1)$-covariant gauge invariant action has the following form in terms of Lorentz covariant field strengths

$$
S^{s, t}=\frac{1}{2 \lambda} \sum_{k, m} \tilde{a}_{k, m}^{s, t} \int_{M^{d}} \epsilon_{c_{1} \ldots c_{d}} e^{c_{5}} \ldots e^{c_{d}} R_{\{2\}}{ }^{c_{1} a(k-1), c_{2} b(m-1)} R_{\{2\}}{ }^{c_{3}}{ }_{a(k-1)}{ }^{, c_{4}}{ }_{b(m-1)},
$$

where

$$
\tilde{a}_{k, m}^{s, t}=a_{k, m}^{s, t} \theta(k-t) \theta(m) \theta(t-m) \theta(s-k-1)
$$

and the coefficients $a_{k, m}^{s, t}$ remain to be determined. This lagrangian generalizes to partially massless fields that introduced in [18] for massless fields $(t=s-1)$.

Let us introduce the inner product

$$
\left\langle\phi_{\{p\}}^{\{k, l\}} \mid \psi_{\{q\}}^{\{k, l\}}\right\rangle=\int_{M^{d}} \epsilon_{c_{1} c_{2} \ldots c_{d}} e^{c_{5}} \wedge \ldots \wedge e^{c_{d}} \wedge \phi_{\{p\}}^{c_{1} a(k-1), c_{2} b(l-1)} \wedge \psi_{\{q\} a(k-1)}^{c_{3}}{ }^{c_{4}}{ }_{b(l-1)},
$$

which is nonzero if the $p$-form $\phi_{\{p\}}^{a(k), b(l)}$ and $q$-form $\psi_{\{q\}}^{a(k), b(l)}$ carry equivalent representations of the Lorentz group (in particular, have the same symmetry type) and are such that $p+q=4$. Then the action (4.30) reads

$$
S^{s, t}=\frac{1}{2 \lambda} \sum_{k, m} \tilde{a}_{k, m}^{s, t}\left\langle R_{\{2\}}^{\{k, m\}} \mid R_{\{2\}}^{\{k, m\}}\right\rangle .
$$


The normalization coefficients $g_{k, l}, G_{k, l}, f_{k, l}$ and $F_{k, l}$ are chosen so (see Appendix C) that the operators $\sigma_{+}^{1,2}$ are conjugated to $\sigma_{-}^{1,2}$ with respect to this product

$$
\left\langle\phi_{\{p\}}^{\{k, l\}}\left|\sigma_{+}^{1,2}\right| \psi_{\{q\}}^{\{k, l\}}\right\rangle=(-)^{p q+p+q}\left\langle\psi_{\{q\}}^{\{k, l\}}\left|\sigma_{-}^{1,2}\right| \phi_{\{p\}}^{\{k, l\}}\right\rangle .
$$

Making use of the Bianchi identities along with

$$
\begin{aligned}
\delta R_{\{2\}}^{\{k, m\}}=D_{0}\left(\delta \omega_{\{1\}}^{\{k, m\}}\right) & =D\left(\delta \omega_{\{1\}}^{\{k, m\}}\right)+\lambda \sigma_{-}^{1}\left(\delta \omega_{\{1\}}^{\{k+1, m\}}\right)+\lambda \sigma_{-}^{2}\left(\delta \omega_{\{1\}}^{\{k, m+1\}}\right)+ \\
& +\lambda \sigma_{+}^{1}\left(\delta \omega_{\{1\}}^{\{k-1, m\}}\right)+\lambda \sigma_{+}^{2}\left(\delta \omega_{\{1\}}^{\{k, m-1\}}\right)
\end{aligned}
$$

the variation of the action amounts to

$$
\begin{aligned}
\delta S^{s, t}=\sum_{k, m} & \left\langle\delta \omega^{\{k, m\}}\left|\sigma_{-}^{1}\right| R^{\{k+1, m\}}\right\rangle\left(\tilde{a}_{k+1, m}^{s, t}-\tilde{a}_{k, m}^{s, t}\right)+ \\
& +\left\langle\delta \omega^{\{k, m\}}\left|\sigma_{-}^{2}\right| R^{\{k, m+1\}}\right\rangle\left(\tilde{a}_{k, m+1}^{s, t}-\tilde{a}_{k, m}^{s, t}\right)+ \\
& +\left\langle\delta \omega^{\{k, m\}}\left|\sigma_{+}^{1}\right| R^{\{k-1, m\}}\right\rangle\left(\tilde{a}_{k-1, m}^{s, t}-\tilde{a}_{k, m}^{s, t}\right)+ \\
& +\left\langle\delta \omega^{\{k, m\}}\left|\sigma_{+}^{2}\right| R^{\{k, m-1\}}\right\rangle\left(\tilde{a}_{k, m-1}^{s, t}-\tilde{a}_{k, m}^{s, t}\right) .
\end{aligned}
$$

The 1-form $W^{A(s-1), B(t)}$ is equivalent to the set of Lorentz 1-forms $\omega^{a(l), b(u)}$ with $l=t, \ldots s-1$ and $u=0,1 \ldots t$. It is easy to see that $W^{A(s-1), B C(t-1)} V_{C(t-1)}$ is equivalent to $\omega^{a(l)}$ and $\omega^{a(l), b}$ with $l=t, \ldots s-1$. As a result, in terms of Lorentz tensors, the extra field decoupling condition (4.13) takes the following simple form

$$
\frac{\delta S^{s, t}}{\delta \omega^{a(k), b(m)}} \equiv 0, \quad k=t, \ldots, s-1, \quad m=2,3, \ldots, t .
$$

It is satisfied with

$$
a_{k, m}^{s, t}=a^{s, t}
$$

For the case of $t>1$ we have

$$
\delta S^{s, t}=a^{s, t} \sum_{k=t}^{k=s-1}\left(\left\langle\delta \omega^{\{k, 0\}}\left|\sigma_{-}^{2}\right| R^{\{k, 1\}}\right\rangle-\left\langle\delta \omega^{\{k, 1\}}\left|\sigma_{+}^{2}\right| R^{\{k, 0\}}\right\rangle\right) .
$$

Upon the rescaling

$$
\begin{aligned}
& \omega^{a(k), b} \rightarrow \frac{1}{\lambda} \omega^{a(k), b}, \quad R^{a(k), b} \rightarrow \frac{1}{\lambda} R^{a(k), b} \\
& S \rightarrow \lambda S
\end{aligned}
$$

the field strengths (4.25) modify to

$$
\begin{gathered}
R_{\{2\}}^{\{k, 0\}}=D\left(\omega_{\{1\}}^{\{k, 0\}}\right)+\sigma_{-}^{1}\left(\omega_{\{1\}}^{\{k, 1\}}\right)+O(\lambda), \\
R_{\{2\}}^{\{k, 1\}}=D\left(\omega_{\{1\}}^{\{k, 1\}}\right)+O(\lambda) .
\end{gathered}
$$


In the flat limit $\lambda \rightarrow 0$, terms that mix different $k$ tend to zero and the field strengths take the form of the flat space field strengths for usual massless fields [18]

$$
\begin{gathered}
R_{\{2\}}^{\{k, 0\}}=D\left(\omega_{\{1\}}^{\{k, 0\}}\right)+\sigma_{-}^{1}\left(\omega_{\{1\}}^{\{k, 1\}}\right), \\
R_{\{2\}}^{\{k, 1\}}=D\left(\omega_{\{1\}}^{\{k, 1\}}\right) .
\end{gathered}
$$

As a result, for $t>1$, the extra field decoupling condition (4.37) guarantees that the flat limit of the action (4.30) is a sum of free actions for massless fields of spins from $t+1$ to $s$ which is in agreement with the construction of Zinoviev [5]. The totally symmetric parts $\omega^{a(k) \mid a}$ of $\omega_{\underline{m}}{ }^{a(k)}, k=t, \ldots, s-1$ identify with the double traceless Fronsdal fields used by Zinoviev.

As it will be explained in more detail in [34], for $\lambda \neq 0$, all fields, that are not pure gauge, can be expressed in terms of derivatives of the dynamical fields $\phi^{a(s)}$,

$\phi^{a(t)}$ and $\phi^{a(t-1)}$ by virtue of constraints $R_{\underline{m n}}^{a(k)}=0, R_{m}^{a(k) m \mid a}=0$, which are particular field equations. As discussed in Subsection 2.3.1, within the approach of Deser and Waldron [4], the constraints result from the divergences of field equations. Note that the reduction of a number of fields compared to those of the approach of Zinoviev does not imply the reduction of a number of degrees of freedom because, as a result of resolution of constrains, the action expressed in terms of the dynamical fields contains higher derivatives for a general partially massless system.

In the special case of $t=1$, where the extra field decoupling condition (4.37) does not apply, the variation of the action (4.30) in terms of the rescaled variables (4.40) is

$$
\begin{aligned}
\delta S^{s, 1}= & \sum_{k=1}^{k=s-1}\left(\left\langle\delta \omega^{\{k, 0\}}\left|\sigma_{-}^{2}\right| R^{\{k, 1\}}\right\rangle-\left\langle\delta \omega^{\{k, 1\}}\left|\sigma_{+}^{2}\right| R^{\{k, 0\}}\right\rangle\right) a_{k, 0}^{s, 1}+ \\
& +\frac{1}{\lambda}\left(\left\langle\delta \omega^{\{k, 1\}}\left|\sigma_{+}^{1}\right| R^{\{k-1,1\}}\right\rangle-\left\langle\delta \omega^{\{k-1,1\}}\left|\sigma_{-}^{1}\right| R^{\{k, 1\}}\right\rangle\right)\left(\tilde{a}_{k-1,0}^{s, 1}-\tilde{a}_{k, 0}^{s, 1}\right) .
\end{aligned}
$$

To get rid of the term singular in the flat limit $\lambda \rightarrow 0$, we set $a_{k, 0}^{s, 1}=a^{s, 1}$ so that (4.38) is still true. The remaining variation reduces in the flat limit to that of the sum of actions for massless fields of spins from 2 to $s$.

\section{Examples}

In this section the proposed approach is illustrated by the simplest examples of spin-2 and spin-3 partially massless fields. 


\section{$5.1 \quad$ Spin-2}

Let us consider the model with $s=2, t=0$. The corresponding gauge field is a 1-form $W_{\{1\}}^{A}$ with the gauge transformation law

$$
\delta W_{\{1\}}^{A}=D_{0} \xi_{\{0\}}^{A} .
$$

The gauge invariant field strength

$$
R_{\{2\}}^{A}=D_{0} W_{\{1\}}^{A}
$$

satisfies the Bianchi identity

$$
D_{0} R_{\{2\}}^{A} \equiv 0 .
$$

The Lorentz reduction of the $(\mathrm{A}) \mathrm{dS}_{d}$ vector $\square$ gives two Lorentz irreducible representations $\bullet \square$, i.e.,

$$
\begin{array}{cc}
\xi_{\{0\}}^{A} \longleftrightarrow \xi=\lambda^{-1} \xi_{\{0\}}^{\bullet}, & \xi^{a}=\xi_{\{0\}}^{a}, \\
W_{\{1\}}^{A} \longleftrightarrow \omega_{\underline{m}}=\lambda^{-1} W_{\{1\}}^{\bullet}, & \omega_{\underline{m}}^{a}=W_{\{1\}}^{a}, \\
R_{\{2\}}^{A} \longleftrightarrow R_{\underline{m n}}=\lambda^{-1} R_{\{2\}}^{\bullet}, & R_{\underline{m n}}^{a}=R_{\{2\}}^{a},
\end{array}
$$

where the rescaling of the first component by $\lambda$ is introduced for the future convenience. The gauge transformation law (5.1) gives

$$
\begin{gathered}
\delta \omega_{\underline{m}}=D_{\underline{m}} \xi-e_{\underline{m} b} \xi^{b}, \\
\delta \omega_{\underline{m}}^{a}=D_{\underline{m}} \xi^{a}+\lambda^{2} e_{\underline{m}}{ }^{a} \xi .
\end{gathered}
$$

From the form of the variation (5.5) it follows that $\omega_{\underline{m}}$ can be gauge fixed to zero

$$
\omega_{\underline{m}}=0
$$

by adjusting the Stueckelberg parameter $\xi^{a}$. Then, from the condition $\delta \omega_{\underline{m}} \equiv 0$ it follows that the leftover gauge symmetry with the parameter $\xi$ requires $\xi^{a}=D^{a} \xi$. Substituting this into the gauge transformation law (5.6) we obtain

$$
\delta \omega_{\underline{m}}^{a}=D_{\underline{m}} D^{a} \xi+\lambda^{2} e_{\underline{m}}^{a} \xi
$$

The field $\omega^{a \mid b}=\omega_{\underline{m}}^{a} e^{b \underline{m}}$ decomposes into $\square \oplus \square \square \oplus \bullet$, i.e., into antisymmetric part $\square=\omega^{[a \mid b]}$ and traceful symmetric part $\square \square \bullet=\omega^{(a \mid b)}$ with the scalar trace field $\bullet=\omega^{c \mid}$. In these terms the gauge transformation law (5.6) reads

$$
\delta \omega^{[a \mid b]}=0,
$$




$$
\delta \omega^{(a \mid b)}=D^{(a} D^{b)} \xi+\lambda^{2} \eta^{a b} \xi
$$

We see that $\omega^{[a \mid b]}$ is gauge invariant while $h^{a b} \equiv \omega^{(a \mid b)}$ transforms as a partially massless spin-2 field (2.13). The field strength (5.2) reduces to

$$
\begin{aligned}
R_{\underline{m n}} & =D_{\underline{m}} \omega_{\underline{n}}-e_{\underline{m} b} \omega_{\underline{n}}^{b}, \\
R_{\underline{m n}}{ }^{a} & =D_{\underline{m}} \omega_{\underline{n}}^{a}+\lambda^{2} e_{\underline{m}}{ }^{a} \omega_{\underline{n}} .
\end{aligned}
$$

In the gauge (5.7) we have

$$
\begin{aligned}
R_{\underline{m n}} & =-e_{\underline{m} b} \omega_{\underline{n}}^{b}, \\
R_{\underline{m n}}{ }^{b} & =D_{\underline{m}} \omega_{\underline{n}}{ }^{a} .
\end{aligned}
$$

The field strength $R_{\underline{m n}}$ has only one irreducible component, $\square$. The field strength $R^{a \mid b c}=R_{\underline{m n}}^{a} e^{b \underline{m}} e^{c \underline{n}}$ decomposes into $\square \oplus \square \bigoplus \square \oplus \square$, with $\square=R^{[a \mid b c]}, \square \oplus \square=$ $R^{(a \mid b) c}$ and $\square=R_{m}^{m \mid}{ }^{a}$.

From (5.12) we see that it is possible to impose the gauge invariant constraint

$$
R_{\underline{m n}}=0
$$

which gets rid of the auxiliary field $\omega^{[a \mid b]}$. Note that analogous constraints express auxiliary fields via derivatives of the other fields in more complicated systems.

The Bianchi identities (5.3) reduce to

$$
\begin{aligned}
& D_{\underline{m}} R_{\underline{n k}}-e_{\underline{m} b} R_{\underline{n k}}^{b} \equiv 0, \\
& D_{\underline{m}} R_{\underline{n k}}^{a}+\lambda^{2} e_{\underline{m}}^{a} R_{\underline{n k}} \equiv 0 .
\end{aligned}
$$

From (5.13) it follows

$$
\begin{aligned}
& e_{\underline{m} b} R_{\underline{n k}}^{b} \equiv 0, \\
& D_{\underline{m}} R_{\underline{n k}}^{a} \equiv 0 .
\end{aligned}
$$

The first identity implies that the totally antisymmetric component

of the field strength $R_{\underline{n k}}{ }^{a}$ is zero. The nonzero part of the field strength $R_{\{2\}}^{A}$ is therefore contained in $C^{[a b], c} \equiv R^{c \mid[a b]}=D^{[a} h^{b] c}$ which is a generalized Weyl tensor. Its trace part is $C_{m}^{a m,}=D \cdot h-D h^{\prime}$. 
Since the gauge invariant tensor $C^{[a b], c}$ contains the first derivatives of the physical field $h^{a a}$, it is possible to impose the following second order equations of motion with the same structure of indices as that of $h^{a a}$

$$
\alpha D_{m} C^{(a m, a)}+\beta D^{(a} C_{m}^{a) m,}+\gamma \eta^{a a} D_{n} C_{m}^{n m,}=0 .
$$

This equation is equivalent to

$$
\alpha\left(2 \square h-D D \cdot h-2 \lambda^{2}\left(d h-\eta h^{\prime}\right)\right)+\beta\left(2 D^{2} h^{\prime}-D D \cdot h\right)+\gamma \eta\left(D^{2} \cdot h-\square h^{\prime}\right)=0 .
$$

By construction, it is gauge invariant under (5.10) for arbitrary $\alpha, \beta$ and $\gamma$. The condition that the flat limit should give the massless field equations in Minkowski space, which is equivalent to the requirement that the massless field gauge symmetry restores in the flat limit, fixes the coefficients up to an overall factor $\alpha=a / 2$, $\beta=a / 2, \gamma=a$. The resulting equations follow from the lagrangian

$$
L=C^{[m n], a} C_{[m n], a}-\frac{1}{2} C_{s}^{[m s],} C_{[m s],},
$$

equivalent to the lagrangian (4.23) at $s=2$. The explicit expression in terms of $h^{a a}$ is

$$
L=\frac{1}{2}(D h)^{2}-(D \cdot h)^{2}-\frac{1}{2}\left(D h^{\prime}\right)^{2}-h^{\prime} D^{2} \cdot h+\frac{d \lambda^{2}}{2} h^{2}-\frac{\lambda^{2}}{2} h^{\prime 2} .
$$

Thus, the $s=2, t=0$ model of Section 2.3 is demonstrated to describe properly the partially massless spin-2 theory of Deser, Waldron [4] and Zinoviev [5]. Note that, in agreement with the general analysis of Section 4, because of the lack of indices carried by the field strength 2 -form in the degenerate case of $t=0$, the lagrangian of this model is formulated in terms of the tensor $C^{[m n], a}$ rather than in terms of the exterior product of the field strength 2 -forms as for $t>0$ systems.

\section{$5.2 \quad$ Spin-3}

The case of spin-3 admits partially massless fields either with $\mathrm{t}=0$ or with $t=1$. The case of $t=0$ is analogous to that considered in Section 5.1 for spin-2 and will not be discussed here. The more interesting case of $s=3, t=1$ partially massless field reveals main features of a general partially massless theory.

The gauge field of this model takes values in the irreducible $(A) d S$ tensor repre-

sentation associated with the Young tableau $\square$. In the manifestly antisymmetric basis it satisfies the conditions $W^{A B, C}=-W^{B A, C}, W^{[A B, C]} \equiv 0$ and $W^{A M, N} \eta_{M N} \equiv 0$ 
(square brackets denote antisymmetrization). The gauge transformation law, field strength and Bianchi identities read

$$
\begin{aligned}
& \delta W_{\{1\}}^{[A B], C}=D_{0} \xi_{\{0\}}^{[A B], C}, \\
& R_{\{2\}}^{[A B], C}=D_{0} W_{\{1\}}^{[A B], C}, \\
& D_{0} R_{\{2\}}^{[A B], C} \equiv 0 .
\end{aligned}
$$

The $(\mathrm{A}) \mathrm{dS}_{d}$ Young tableau $\square$ decomposes into the following irreps of $o(d-1,1)$

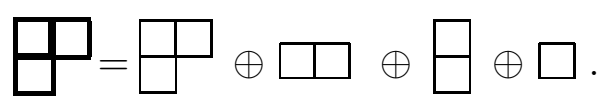

Let us introduce the following notation for the gauge parameters

$$
\begin{aligned}
& \xi^{a}=\xi_{\{0\}}^{a \bullet \bullet}, \\
& \xi^{a b}=\xi_{\{0\}}^{[a \bullet, b]}, \\
& \xi^{a, c}=\xi_{\{0\}}^{(a \bullet, c)}, \\
& \xi^{a b, c}=\xi_{\{0\}}^{a b, c}+\frac{1}{d-1}\left(\xi^{a} \eta^{b c}-\xi^{b} \eta^{a c}\right) .
\end{aligned}
$$

The fields $\omega_{\underline{m}}^{a}, \omega_{\underline{m}}^{a b}, \omega_{\underline{m}}^{a, c}, \omega_{\underline{m}}^{a b, c}$ and the corresponding field strengths are defined analogously. In these terms the gauge transformation laws read

$$
\begin{aligned}
\delta{\underline{\omega_{m}}}^{a} & =D_{\underline{m}} \xi^{a}+3 \lambda e_{\underline{m} m} \xi^{a m}+\lambda e_{\underline{m} m} \xi^{a, m} \\
\delta \underline{\omega}_{\underline{m}}^{a b} & =D_{\underline{m}} \xi^{a b}+\frac{\lambda d}{d-1} e_{\underline{m}}^{[a} \xi^{b]}+\frac{1}{2} \lambda e_{\underline{m} m} \xi^{a b, m} \\
\delta \underline{\omega}_{\underline{m}}^{a, b} & =D_{\underline{m}} \xi^{a, b}-\frac{\lambda d}{d-1} e_{\underline{m}}^{(a} \xi^{b)}+\frac{\lambda}{d-1} e_{\underline{m} m} \xi^{m} \eta^{a b}+\lambda e_{\underline{m} m} \xi^{(a m, b)}, \\
\delta \underline{\omega}_{\underline{m}}^{a b, c} & =D_{\underline{m}} \xi^{a b, c}+\lambda e_{\underline{m}}^{a} \xi^{b c}+\lambda e_{\underline{m}}^{a} \xi^{b, c}-\lambda e_{\underline{m}}^{b} \xi^{a c}-\lambda e_{\underline{m}}^{b} \xi^{a, c}-2 \lambda e_{\underline{m}}^{c} \xi^{a b}+ \\
& +\frac{\lambda}{d-1} \eta^{b c} e_{\underline{m} m}\left(3 \xi^{a m}+\xi^{a, m}\right)-\frac{\lambda}{d-1} \eta^{a c} \lambda e_{\underline{m} m}\left(3 \xi^{b m}+\xi^{b, m}\right) .
\end{aligned}
$$

The decomposition of the fields into irreps of the Lorentz algebra $o(d-1,1)$, that acts diagonally on the fiber indices $a, b, c \ldots$ and the base indices $\underline{m}, \underline{n} \ldots$, is as follows

$$
\left\{\begin{array}{l}
\omega_{\underline{m}^{a}}{ }^{a} \square \oplus \square \oplus \bullet, \\
\square \square \bullet=\omega^{(a \mid b)}, \square=\omega^{[a \mid b]}, \chi \equiv \bullet=\omega_{m}^{m \mid},
\end{array}\right.
$$




$$
\left\{\begin{array}{l}
\omega_{\underline{m}^{a}}{ }^{a, c}=\square_{1} \oplus \square \square \square \oplus \square, \\
\square_{1}=\Pi\left[\omega^{a, b \mid c}-\omega^{c,(a \mid b)}\right], \square \square \square=\Pi\left[\phi^{a b c} \equiv \omega^{(a, b \mid c)}\right], \psi_{1}^{a} \frac{3}{2} \equiv \square^{1}=\omega^{a, m \mid}, \quad \phi^{\prime}=\psi_{1},
\end{array}\right.
$$

$$
\begin{aligned}
& \left\{\begin{array}{l}
\omega_{\underline{m}}^{a b}=\square_{2} \oplus \square \oplus \square^{2}, \\
\square \square_{2}=\Pi\left[\omega^{a b \mid c}-\omega^{[a b \mid c]}\right], \square=\omega^{[a b \mid c]}, \psi_{2}{ }^{a} \frac{1}{2} \equiv \square^{2}=\omega_{m}^{a m \mid},
\end{array}\right.
\end{aligned}
$$

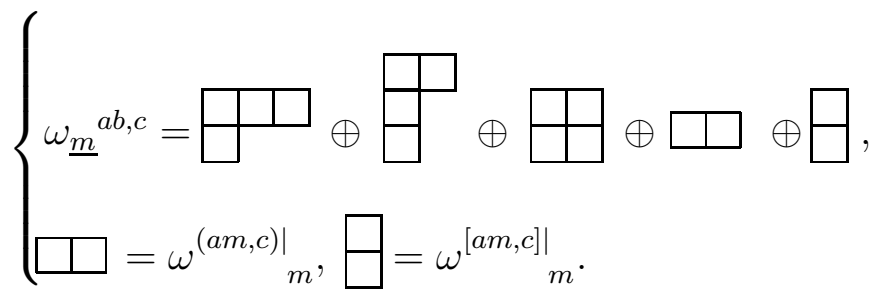

Explicit expressions are given here for those components, which appear in the subsequent analysis; the labels 1,2 distinguish between different components of the same symmetry type.

From (15.23) it follows that the components $\square \square$ and $\square$ of $\omega_{\underline{m}}^{a}$ and $\square_{1}$ of $\omega_{\underline{m}}^{a, c}$ can be gauge fixed to zero by the Stueckelberg gauge parameters $\xi^{a, c}, \xi^{a b}$ and $\xi^{a b, c}$. We therefore set

$$
\omega^{[a \mid b]}=0, \quad \Pi\left[\omega^{(a \mid b)}\right]=0, \quad \Pi\left[\left(\omega^{a, b \mid c}-\omega^{c,(a \mid b)}\right)\right]=0 .
$$

This gauge is not complete. In the leftover gauge transformations, that leave invariant the gauge conditions, the parameters $\xi^{a, c}, \xi^{a b}$ and $\xi^{a b, c}$ are expressed in terms of derivatives of $\xi^{a}$, which is the only non-Stueckelberg gauge parameter. The gauge transformation law of the component $\phi^{a a a}$ has the form (2.21) of the spin-3 partially massless theory, i.e., $\phi^{a a a}$ identifies with the dynamical field $\phi$ of Deser and Waldron. 
The field strengths have the form

$$
\begin{aligned}
& R_{\underline{m n}}^{a}=D_{\underline{m}} \omega_{\underline{n}}^{a}+3 \lambda e_{\underline{m} m} \omega_{\underline{n}}^{a m}+\lambda e_{\underline{m} m} \omega_{\underline{n}}^{a, m}, \\
& R_{\underline{m n}}^{a b}=D_{\underline{m}} \omega_{\underline{n}}^{a b}+\frac{d \lambda}{d-1} e_{\underline{m}}^{[a} \omega_{\underline{n}}^{b]}+\frac{1}{2} \lambda e_{\underline{m} m} \omega_{\underline{n}}^{a b, m}, \\
& R_{\underline{m n}}{ }^{a, b}=D_{\underline{m}} \omega_{\underline{n}}^{a, b}-\frac{d \lambda}{d-1} e_{\underline{m}}^{(a} \omega_{\underline{n}}^{b)}+\frac{\lambda}{d-1} e_{\underline{m} m} \omega_{\underline{n}}^{m} \eta^{a b}+\lambda e_{\underline{m} m} \omega_{\underline{n}}^{(a m, b)},
\end{aligned}
$$

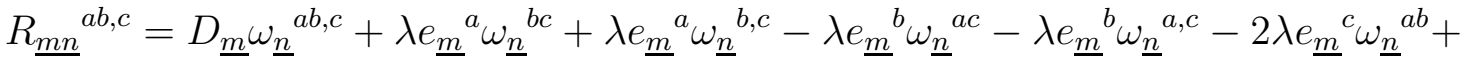

$$
\begin{aligned}
& +\frac{\lambda}{d-1} \eta^{b c} e_{\underline{m} m}\left(3 \omega_{\underline{n}}^{a m}+\omega_{\underline{n}}^{a, m}\right)-\frac{\lambda}{d-1} \eta^{a c} e_{\underline{m} m}\left(3 \omega_{\underline{n}}^{b m}+\omega_{\underline{n}}^{b, m}\right) \text {. }
\end{aligned}
$$

Let us consider which gauge invariant conditions can be imposed on the fields by setting to zero some of components of the field strengths. These may include either constraints, that express auxiliary fields in terms of derivatives of the dynamical fields, or impose differential field equations on the dynamical fields.

The simplest constraint is $R_{\underline{m n}}{ }^{a}=0$. Taking into account the gauge conditions (5.28), it implies

$$
\begin{gathered}
\square=\omega^{[a b \mid c]}=0, \\
\square{ }_{2}=\Pi\left[\omega^{a b \mid c}-\omega^{[a b \mid c]}\right]=0, \\
\frac{d-1}{d} D^{a} \chi-\frac{3}{2} \psi_{2}{ }^{a}+\frac{3}{2} \psi_{1}{ }^{a}=0 .
\end{gathered}
$$

The constraint (5.32) can be used to express the auxiliary field ${\psi_{2}}^{a}$ in terms of $\psi_{1}{ }^{a}$ and the first derivative of the scalar field $\chi$. The constraint (5.31) in the gauge (5.28) implies that the hook-symmetry parts of $\omega^{a b}$ and $\omega^{a, b}$ are zero. As a result one is left with traces $\chi,{\psi_{2}}^{a}$ of $\omega^{a}, \omega^{a b}$, with the totally symmetric component $\phi^{a a a}$ of $\omega^{a, b}$, with its trace $\psi_{1}^{a}$ and the field $\omega^{a b, c}$, which has not been yet considered.

Let us now impose the condition $R_{\underline{m n}}{ }^{a, b}=0$. It implies that

$$
\begin{gathered}
\square \\
\square=\Pi\left[R^{a,[b \mid b b]}\right]=\Pi\left[\omega^{[b b, a \mid b]}\right]=0, \\
\omega^{(a m, a \mid a)}=-D^{m} \phi^{a a a}+D^{(a} \omega^{a, a) \mid m},
\end{gathered}
$$

expressing the auxiliary fields $\omega^{(a m, a \mid a)}=\square \square \oplus \square \square \oplus \square$ in terms of the first derivatives of $\phi^{a a a}$ and setting the component $\stackrel{\square}{\square}=\omega^{[a a, b \mid a]}$ to zero. 
The condition that the projection of $R^{[a b], c}$ to the components of the symmetry $\square \square$ and $\square$ is zero (i.e., $R_{m}^{m(a, a \mid a)}=0$ and $R^{s t, a \mid}{ }_{s t}=0$ ) gives the following equation

$$
\square \phi-D D \cdot \phi+\frac{1}{2} D D \psi_{1}-3 \lambda^{2} \phi+\eta \lambda^{2} \psi_{1}-\eta \lambda^{2} \frac{d-2}{3 d} D \chi=0,
$$

which is the dynamical equation on $\phi$ and its trace $\psi_{1}$.

The condition $R_{\underline{m n}}^{a b}=0$ in the gauge (5.28) sets to zero the component $\square$ of $\omega^{a b, c}$ and $\Pi\left[R_{m}^{(a m \mid a)}\right]=0$ implies

$$
\Pi\left[D^{(a} \psi_{1}^{a)}-D_{m} \phi^{m a a}+\frac{d-2}{3 d \lambda} D^{a} D^{a} \chi\right]=0,
$$

while $R_{s t}{ }^{s t}=0$ imposes the equation

$$
D_{m} \psi_{2}^{m}+\lambda d \chi=0
$$

Using (5.32) to exclude $\psi_{2}{ }^{a}$, we obtain

$$
\frac{2(d-1)}{3 d} \square \chi+\lambda D_{m} \psi_{1}^{m}+d \lambda^{2} \chi=0
$$

which is the equation on $\chi$.

As a result, one has the dynamical equations (5.35), (5.38) on $\phi^{a a a}$, its trace $\psi_{1}^{a}$ and $\chi$, plus one differential constraint (5.36), which corresponds to equation (2.17) of the approach of Deser and Waldron.

Consider a $(\mathrm{A}) \mathrm{dS}_{d}$-covariant, gauge invariant action of the form

$$
S=\frac{1}{2 \lambda} \int_{M^{d}}\left(a_{1} R_{\{2\}}^{\left[A_{1} A_{2}\right], B} R_{\{2\}}^{\left[A_{3} A_{4}\right],}{ }_{B}+a_{2} R_{\{2\}}^{\left[A_{1} A_{2}\right], C} R_{\{2\}}^{\left[A_{3} A_{4}\right], C} V_{C(2)}\right) \epsilon_{A_{1} \ldots A_{d+1}} V^{A_{5}} E^{A_{6}} \ldots E^{A_{d+1}}
$$

In terms of Lorentz components rescaled according to (4.40), its variation turns out to be

$$
\begin{aligned}
& \delta S=\int_{M^{d}} \alpha\left(\delta \omega^{\left[a_{1} a_{2}\right]} R^{a_{3}}+\delta \omega^{a_{1}} R^{\left[a_{2} a_{3}\right]}\right)+ \\
&+ \beta\left(\delta \omega^{\left[a_{1} a_{2}\right], b} R^{a_{3},}{ }_{b}+\delta \omega^{a_{1}, b} R^{\left[a_{2} a_{3}\right],}{ }_{b}\right)+ \\
&+\frac{1}{\lambda} \gamma\left(\delta \omega^{\left[a_{1} a_{2}\right], b} R^{a_{3}}{ }_{b}+\delta \omega^{\left[a_{1} b\right]} R^{\left[a_{2} a_{3}\right],}{ }_{b}\right) \varepsilon_{a_{1} \ldots a_{d}} h^{a_{4}} \ldots h^{a_{d}},
\end{aligned}
$$

where

$$
\alpha=\frac{2 d}{d-1}\left(a_{1}+a_{2}\right), \quad \beta=a_{1}, \quad \gamma=a_{1}-\frac{2}{d-3} a_{2} .
$$


To have correct flat limit we set $\gamma=0$. As a result $a_{2}=\frac{d-3}{2} a_{1}$, which is in accordance with the general formula (4.19). The resulting action gives the following equations of motion

$$
\begin{gathered}
\frac{\delta S}{\delta \omega_{\underline{m}}^{[a b]}}=0 \Longleftrightarrow R_{\underline{m n}}{ }^{a}=0, \\
\frac{\delta S}{\delta \chi}=0 \Longleftrightarrow R_{s t}{ }^{s t}=0, \\
\frac{\delta S}{\delta \omega_{\underline{m}}{ }^{[a b], c}}=0 \Longleftrightarrow R_{m n}{ }^{a, c}=0, \\
\frac{\delta S}{\delta \phi^{a a a}}=0 \Longleftrightarrow R_{m}^{(a m, a \mid a)}=0 .
\end{gathered}
$$

These equations are equivalent to the conditions (5.30)-(5.38) on the field strengths, which have been just shown to describe properly the spin-3 partially massless theory of depth one.

\section{Discussion}

In this paper, partially massless fields in $(A) d S$ space studied in [1]-8] are shown to admit a natural formulation in terms of gauge 1 -forms that take values in irreducible tensor representations of the algebra $(o(d, 1)) o(d-1,2)$, described by the various tworow Young tableaux. The case of rectangular two-row Young tableaux corresponds to the massless fields [19]. Different non-rectangular Young tableaux correspond to different partially massless fields. The manifestly gauge invariant free lagrangians are bilinears of gauge invariant field strengths.

One of the applications of the obtained results is that they impose strong restrictions on possible HS algebras. Actually, since partially massless fields in $A d S_{d}$ correspond to non-unitary representations of $o(d-1,2)$, for a HS theory in $A d S_{d}$ to be unitary, it should be free of gauge fields associated with partially massless fields. This means that a HS algebra $g$ considered as a $o(d-1,2)$-module with respect to its adjoint action in $g$ should not contain submodules described by the corresponding Young tableaux. This condition is indeed satisfied in the case of HS algebras underlying HS theories of totally symmetric fields [14]. The simplest example of a HS algebra, which is associated with the supersymmetric extension of the theory of symmetric HS fields studied in [14], also satisfies this condition. It should be true, however, for any other unitary HS theory including those that contain generic mixed symmetry fields [35], [36], [37, thus imposing nontrivial restrictions on a structure of a HS algebra and, therefore, on the spectra of HS gauge fields that may appear in consistent HS gauge theories. 
It would be interesting to extend the results of this paper to the case of general mixed symmetry partially massless bosonic and fermionic fields, originally discussed in [37]. Taking into account the results of [20], where it has been shown that massless fields of a general symmetry type are described by $p$-form gauge fields that carry a representation of $o(d-1,2)$ described by a Young tableau with at least $p+1$ cells in the shortest column, it is natural to conjecture that partially massless fields as well as the "nonunitary massless fields" with the gauge parameters ruled out in [10] are described in terms of $p$-form gauge fields that are $o(d-1,2)$-modules depicted by Young tableaux with at most $p$ cells in the shortest column. The analysis of this problem, that we leave for a future publication, will provide the full list of unitary and non-unitary free HS models described by gauge $p$-form fields that take values in various finite-dimensional $o(d-1,2)$-modules.

\section{Acknowledgements}

The authors acknowledge with gratitude the collaboration of V.Didenko at the early stage of this work. E.S. is grateful to K.B.Alkalaev for useful discussions. The work was supported in part by grants RFBR No. 05-02-17654, LSS No. 1578.2003-2 and INTAS No. 03-51-6346. The work of E.S. was supported in part by the scholarship of Dynasty Foundation. 


\section{A Appendix A: Notation}

\section{A.1 Index conventions}

Latin indices $A, B, \ldots=0,1, \ldots, d$ are vector indices of the isometry algebra $o(d-1,2)$ or $o(d, 1)$. Indices $a, b, \ldots=0, \ldots, d-1$ are vector indices of the $d$-dimensional Lorentz algebra $o(d-1,1)$. For definiteness, we consider the $(\mathrm{A}) \mathrm{dS}_{d}$ case of $o(d-1,2)$. For the decomposition of irreps of $o(d-1,2)$ into irreps of $o(d-1,1)$ we use notations $A=\{a, \bullet\}$, where $\bullet$ denotes the extra value of the $o(d-1,2)$ vector index compared to the $o(d-1,1)$ vector index.

Underlined Latin indices $\underline{m}, \underline{n}, \underline{k}, \ldots=0,1, \ldots d-1$ are the indices of differential forms in the space-time base manifold. $d=d x \underline{\underline{m}} \partial_{\underline{m}}$ is the de Rham differential. To simplify formulae we often omit the exterior differentials $d x^{\underline{m}}$ and the wedge product symbol $\wedge$. The rank of a differential form is indicated as a braced subscript. For example, $\omega_{\{r\}}$ denotes a $r$-form $\omega_{\underline{m}_{1} \ldots \underline{m}_{r}}$.

$\eta_{A B}$ and $\eta_{a b}$ are, respectively, $o(d-1,2)$ and $o(d-1,1)$ invariant metrics. We use the mostly minus signature, i.e., $\eta_{A B}=\operatorname{diag}(\overbrace{+-\ldots-+}^{d+1})$ and $\eta_{a b}=\operatorname{diag}(\overbrace{+-\ldots-}^{d})$. $\epsilon_{A_{1} \ldots A_{d+1}}$ and $\epsilon_{a_{1} \ldots a_{d}}$ are Levi-Civita tensors of the algebras $o(d-1,2)$ and $o(d-1,1)$, respectively. $e_{\underline{m}}^{a}$ is the vielbein 1 -form. The metric tensor $g_{\underline{m n}}=e_{\underline{m}}^{a} e_{\underline{n}}^{b} \eta_{a b}$ is required to be nondegenerate. $\lambda^{-1}$ is the radius of $(A) d S_{d}$.

To simplify formulae we use multi-index notation. Namely, a group of indices of any kind (in particular, vector indices of the algebras $o(d-1,2)$ and $o(d-1,1)$ ) in which some tensor $C^{a_{1} a_{2} \ldots a_{n}, b, c \ldots}$ is symmetric, that is

$$
C^{a_{1} a_{2} \ldots a_{i} \ldots a_{j} \ldots a_{n}, b, c, \ldots}=C^{a_{1} a_{2} \ldots a_{j} \ldots a_{i} \ldots a_{n}, b, c, \ldots}
$$

is denoted $C^{a(n), b, c, \ldots} \equiv C^{\left(a_{1} a_{2} \ldots a_{n}\right), b, c, \ldots}$. The similar notation $C^{a[n], \ldots,} \equiv C^{[a a \ldots a], \ldots,}$ is used for antisymmetric indices. More generally, symmetrized upper or lower indices are denoted by the same letter. We use the normalization in which the symmetrization operator $\widehat{\mathbf{S}}$ is a projector $\widehat{\mathbf{S}}^{2}=\widehat{\mathbf{S}}$. For example, for any vector $V^{a}$ and a totally symmetric rank- $n$ tensor $C^{a(n)}$

$$
V^{a} C^{a(n)}=\frac{1}{n+1} \sum_{i=1}^{i=n+1} V^{a_{i}} C^{a_{1} \ldots \widehat{a_{i}} \ldots a_{n+1}},
$$

where the hatted index has to be omitted. 


\section{A.2 Condensed Notation}

The traceless part of a tensor $T$ is denoted $\boldsymbol{\Pi}[T]$. For example, for a rank-2 tensor, $\phi_{a b}$

$$
\Pi\left[\phi_{a b}\right]=\phi_{a b}-\frac{1}{d} \eta_{a b} \phi_{c}^{c}
$$

A tensor field that has the symmetry properties depicted by the Young tableau composed of rows of lengths $n_{1}, \ldots, n_{p}$ is denoted $\phi^{\left\{n_{1}, \ldots, n_{p}\right\}}$.

The following notation is often used for a totally symmetric rank-s tensor field $\phi_{\underline{m}_{1} \ldots \underline{m}_{s}}(x)$ and its derivatives

$$
\begin{aligned}
& \phi^{\{s\}} \equiv \phi_{\underline{m}_{1} \ldots \underline{m}_{s}}(x) \\
& \partial \phi^{\{s\}} \equiv s \partial_{\left(\underline{m}_{1}\right.} \phi_{\left.\underline{m}_{2} \ldots \underline{m}_{s+1}\right)}(x), \\
& \square \phi^{\{s\}} \equiv \partial^{\underline{m}} \partial_{\underline{m}} \phi^{\{s\}}, \\
& \partial \cdot \phi^{\{s\}} \equiv \partial^{\underline{m}} \phi_{\underline{m m}_{2} \ldots \underline{m}_{s}}(x), \\
& \partial^{2} \cdot \phi^{\{s\}} \equiv \partial \cdot\left(\partial \cdot \phi^{\{s\}}\right), \\
& \phi^{\{s\}^{\prime}} \equiv \phi^{\underline{m}}{\underline{m m_{3}}}_{3} \ldots \underline{m}_{s}(x), \\
& \left(\partial \cdot \phi^{\{s\}}\right)^{2} \equiv \partial^{\underline{n}} \phi_{\underline{n m}_{2} \cdots \underline{m}_{s}}(x) \partial_{\underline{k}} \phi^{\underline{k m_{2}} \cdots \underline{m}_{s}}(x), \\
& \eta \phi^{\{s\}} \equiv \frac{(s+2)(s+1)}{2} \eta_{\left(\underline{m}_{1} \underline{m}_{2}\right.} \phi_{\left.\underline{m}_{3} \ldots \underline{m}_{s+2}\right)} .
\end{aligned}
$$

\section{B Appendix B: Young tableaux}

A Young tableau $\left\{n_{i}\right\}(i=1,2, \ldots, p)$ is a set of positive integers such that $n_{i} \geq$ $n_{j}>0, i>j$. It can be depicted as a set of rows of length $n_{i}$.

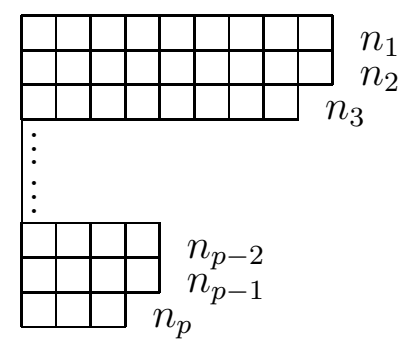

Each box corresponds to an index of a tensor

$$
C^{A_{1} A_{2} \ldots A_{n_{1}}, B_{1} B_{2} \ldots B_{n_{2}}, \ldots \ldots, Z_{1} Z_{2} \ldots Z_{n_{p}}}
$$

that has the following properties 
1. The tensor $C^{A_{1} A_{2} \ldots A_{n_{1}}, B_{1} B_{2} \ldots B_{n_{2}}, \ldots \ldots, Z_{1} Z_{2} \ldots Z_{n_{p}}}$ is symmetric in indices $A_{1} \ldots A_{n_{1}}$, $B_{1} \ldots B_{n_{2}}$ and so forth, that is, in multi-index notation, it is $C^{A\left(n_{1}\right), B\left(n_{2}\right), \ldots, Z\left(n_{p}\right)}$.

2. Symmetrization of all indices of any group with an index of a subsequent groups is identically zero

$$
C^{A\left(n_{1}\right), \ldots,\left(F\left(n_{k}\right), \ldots, F\right) N\left(n_{m}-1\right), \ldots, Z\left(n_{p}\right)}=0 .
$$

A scalar is symbolized by $\bullet$.

Different symmetry types of tensors are characterized by Young tableaux. One can use different bases in the vector space of tensors of a given type. The one we mostly use in this paper is that with explicit symmetrizations. Alternatively, one can use the antisymmetric basis, which is used in particular in Section 5.2 of this paper. (For more detail, see e.g. [31.)

Since the metric and the Levi-Civita tensor $\epsilon_{A_{1} \ldots A_{d}}$ are invariant tensors of the algebra $o(r, q)$, to single out its irreps the tracelessness condition, the condition that the height of any column does not exceed $[d / 2]$ have to be imposed. For even $d$ and appropriate signature the (anti)selfduality condition has to be imposed on the tensor representations associated with Young tableaux that have at least one column of height $d / 2$.

The decomposition of the tensor product of any irrep of $s l_{d}$ with its vector representation admits a natural description in terms of Young tableaux. Namely, it contains all tableaux that can be obtained by adding a box to a given Young tableau. For example,

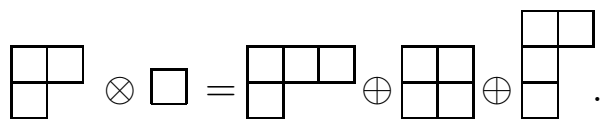

In the case of $o(r, q)$, Young tableaux are associated with traceless tensors of one or another symmetry type. The tensor product decomposition results from either adding or cutting one box in various ways. The cutted diagrams result from traces between the tensor product factors. For example, the $o(r, q)$ decomposition analogous to (B.3) is

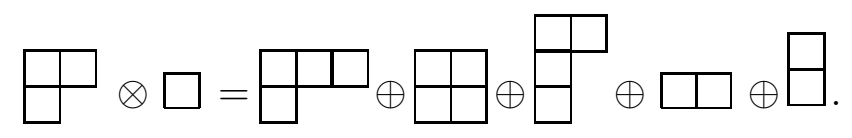

To decompose an irrep of $o(d-1,2)$ into irreps of its subalgebra $o(d-1,1)$ it is convenient to identify the latter with the stability subalgebra of some nonzero 
time-like vector $V^{A}$. The mnemonic is that to obtain a set of tableaux of $o(d-1,1)$ one has to cut off boxes of the Young tableau of $o(d-1,2)$ in all possible ways so that no two boxes were cut off in the same column. For example, for the $o(d-1,2)$

tableau $\square \quad$ we obtain the following set of $o(d-1,1)$ tableaux

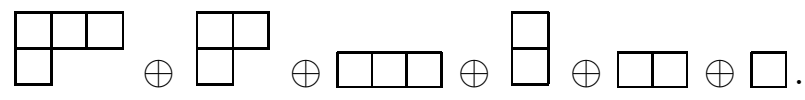

In this paper, fields, gauge parameters and field strengths are $p$-forms with fiber indices in finite-dimensional modules of $o(d-1,1)$ or $o(d-1,2)$ described by various Young tableaux. Replacing the form index of a 1-form by the fiber one with the help of the vielbein $e_{\underline{m}}^{a}$ (e.g., $\omega^{a(3), b \mid c}=\omega_{\underline{m}}{ }^{a(3), b} e^{\underline{m} \mid c}$ ), the resulting fiber tensor decomposes into irreps of $o(d-1,1)$ according to the general rule for the tensor product with the vector representation. For instance, one obtains for $\omega^{a(3), b \mid c}$

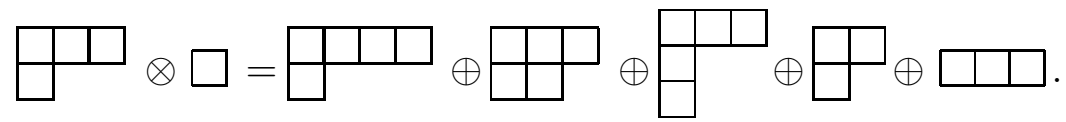

In particular, here $\square \square \square$ corresponds to $\omega_{\underline{m}}^{a(3), b} e^{\underline{m}} b$.

\section{Appendix C: Coefficients}

The final result is

$$
\begin{gathered}
g(k, m)=\sqrt{h(k, m) \frac{k+1}{k(d+k-2)}}, \\
G(k, m)=\sqrt{H(k, m) \frac{k-m}{(d+m-3)(k-m+1)}}, \\
f(k, m)=\sqrt{h(k-1, m) \frac{(k-1)(d+k-3)}{k}}, \\
F(k, m)=\sqrt{H(k, m-1) \frac{(d+m-4)(k-m+2)}{k-m+1}},
\end{gathered}
$$

where

$$
\begin{array}{r}
h(k, m)=\frac{(k-t+1)(d+k+t-2)(s-k-1)(d+s+k-2)}{(k-m+1)(d+k+m-2)(d+2 k)}, \\
H(k, m)=\frac{(s-m)(d+s+m-3)(t-m)(d+t+m-3)}{(k-m+1)(d+u+m-2)(d+2 m-2)} .
\end{array}
$$


These coefficients satisfy the following relations

$$
\begin{array}{r}
f(k+1, m)=\frac{(d+k-2) k}{(k+1)} g(k, m), \\
F(k, m+1)=\frac{(d+m-3)(k-m+1)}{(k-m)} G(k, m)
\end{array}
$$

which insure that the operators $\sigma_{+}^{1,2}$ are conjugated to $\sigma_{-}^{1,2}$ with respect to the scalar product (4.32). Also note that

$$
\begin{gathered}
h(k, m)=f(k+1, m) g(k, m), \\
H(k, m)=F(k, m+1) G(k, m) .
\end{gathered}
$$




\section{References}

[1] S. Deser and R. I. Nepomechie, Phys. Lett. B 132 (1983) 321; Annals Phys. 154 (1984) 396.

[2] A. Higuchi, YTP-85-22; Nucl. Phys. B 282, 397 (1987); J. Math. Phys. 28, 1553 (1987) [Erratum-ibid. 43, 6385 (2002)]; Class. Quant. Grav. 6, 397 (1989); Nucl. Phys. B 325, 745 (1989).

[3] I. Bengtsson, J. Math. Phys. 36, 5805 (1995) arXiv:gr-qc/9411057.

[4] S. Deser and A. Waldron, Phys. Rev. Lett. 87, 031601 (2001) arXiv:hep-th/0102166; Nucl. Phys. B 607 (2001) 577 arXiv:hep-th/0103198; Phys. Lett. B 508, 347 (2001) |arXiv:hep-th/0103255]; Phys. Lett. B 513, 137 (2001) arXiv:hep-th/0105181; Nucl. Phys. B 662 (2003) 379 arXiv:hep-th/0301068; "Conformal invariance of partially massless higher spins", arXiv:hep-th/0408155.

[5] Y. M. Zinoviev, "On massive high spin particles in (A)dS", arXiv:hep-th/0108192.

[6] I. L. Buchbinder, D. M. Gitman and V. D. Pershin, Phys. Lett. B 492, 161 (2000) arXiv:hep-th/0006144.

[7] K. Hallowell and A. Waldron, Nucl. Phys. B 724, 453 (2005) arXiv:hep-th/0505255.

[8] L. Dolan, C. R. Nappi and E. Witten, JHEP 0110, 016 (2001) arXiv:hep-th/0109096.

[9] N. T. Evans, J. Math. Phys. 8, 170 (1967).

[10] R.R. Metsaev, Phys. Lett. B 354 (1995) 78; "Arbitrary spin massless bosonic fields in d-dimensional anti-de Sitter space," arXiv:hep-th/9810231.

[11] E. Angelopoulos and M. Laoues, Rev. Math. Phys. 10, 271 (1998) arXiv:hep-th/9806100.

[12] L. Brink, R. R. Metsaev and M. A. Vasiliev, Nucl. Phys. B 586, 183 (2000) arXiv:hep-th/0005136.

[13] S. Ferrara and C. Fronsdal, "Conformal fields in higher dimensions," arXiv:hep-th/0006009. 
[14] M. A. Vasiliev, JHEP 0412, 046 (2004) arXiv:hep-th/0404124].

[15] F. A. Dolan, "Character formulae and partition functions in higher dimensional conformal field theory," arXiv:hep-th/0508031.

[16] M. A. Vasiliev, Yad. Fiz. 32, 855 (1980).

[17] M. A. Vasiliev, Fortsch. Phys. 35, 741 (1987) [Yad. Fiz. 45, 1784 (1987)].

[18] V. E. Lopatin and M. A. Vasiliev, Mod. Phys. Lett. A 3, 257 (1988).

[19] M. A. Vasiliev, Nucl. Phys. B 616 (2001) 106 [Erratum-ibid. B 652 (2003) 407] arXiv:hep-th/0106200].

[20] K. B. Alkalaev, O. V. Shaynkman and M. A. Vasiliev, Nucl. Phys. B 692 (2004) 363 [arXiv:hep-th/0311164].

[21] Dirac, P. A. M., Royal Society of London Proceedings Series A, 155, 447, 1936.

[22] M. Fierz and W. Pauli, Proc. Roy. Soc. Lond. A 173 (1939) 211.

[23] L. P. S. Singh and C. R. Hagen, Phys. Rev. D 9 (1974) 898.

[24] M. Bianchi and F. Riccioni, "Massive higher spins and holography," arXiv:hep-th/0601071.

[25] I. L. Buchbinder and V. A. Krykhtin, Nucl. Phys. B 727, 537 (2005) arXiv:hep-th/0505092].

[26] I. L. Buchbinder and V. A. Krykhtin, "BRST approach to higher spin field theories," arXiv:hep-th/0511276.

[27] C. Fronsdal, Phys. Rev. D 18 (1978) 3624.

[28] I. L. Buchbinder, V. A. Krykhtin and A. Pashnev, Nucl. Phys. B 711, 367 (2005) arXiv:hep-th/0410215|.

[29] D. Francia and A. Sagnotti, Phys. Lett. B 543, 303 (2002) arXiv:hep-th/0207002; Class. Quant. Grav. 20, S473 (2003) arXiv:hep-th/0212185; Phys. Lett. B 624, 93 (2005) arXiv:hep-th/0507144.

[30] S. W. MacDowell and F. Mansouri, Phys. Rev. Lett. 38, 739 (1977) [Erratumibid. 38, 1376 (1977)]. 
[31] X. Bekaert, S. Cnockaert, C. Iazeolla and M. A. Vasiliev, "Nonlinear higher spin theories in various dimensions", arXiv:hep-th/0503128.

[32] K. S. Stelle and P. C. West, Phys. Rev. D 21 (1980) 1466.

[33] M. A. Vasiliev, 'Higher spin gauge theories: Star-product and AdS space," arXiv:hep-th/9910096; Comptes Rendus Physique 5, 1101 (2004) |arXiv:hep-th/0409260];

E. Sezgin and P. Sundell, Nucl. Phys. B 644, 303 (2002) [Erratum-ibid. B 660, 403 (2003)] arXiv:hep-th/0205131;

D. Sorokin, AIP Conf. Proc. 767, 172 (2005) arXiv:hep-th/0405069;;

N. Bouatta, G. Compere and A. Sagnotti, "An introduction to free higher-spin fields," arXiv:hep-th/0409068.

[34] E. D. Skvortsov, M. A. Vasiliev, in preparation.

[35] K. B. Alkalaev, O. V. Shaynkman and M. A. Vasiliev, JHEP 0508, 069 (2005) arXiv:hep-th/0501108.

[36] T. Curtright and P. G. O. Freund, Nucl. Phys. B 172, 413 (1980);

T. Curtright, Phys. Lett. B 165, 304 (1985);

C. S. Aulakh, I. G. Koh and S. Ouvry, Phys. Lett. B 173, 284 (1986);

J. M. F. Labastida and T. R. Morris, Phys. Lett. B 180, 101 (1986). J. M. F. Labastida, Phys. Rev. Lett. 58, 531 (1987); Nucl. Phys. B 322, 185 (1989);

M. Dubois-Violette and M. Henneaux, Lett. Math. Phys. 49, 245 (1999) arXiv:math.qa/9907135; Commun. Math. Phys. 226, 393 (2002) arXiv:math.qa/0110088;

C. Burdik, A. Pashnev and M. Tsulaia, Mod. Phys. Lett. A 16, 731 (2001) arXiv:hep-th/0101201;

P. de Medeiros and C. Hull, JHEP 0305, 019 (2003) arXiv:hep-th/0303036;

P. de Medeiros, Class. Quant. Grav. 21, 2571 (2004) arXiv:hep-th/0311254;

X. Bekaert and N. Boulanger, Commun. Math. Phys. 245, 27 (2004) arXiv:hep-th/0208058;

C. C. Ciobirca, E. M. Cioroianu and S. O. Saliu, Int. J. Mod. Phys. A 19, 4579 (2004) arXiv:hep-th/0403017;

R. R. Metsaev, Class. Quant. Grav. 22, 2777 (2005) arXiv:hep-th/0412311; 
K. B. Alkalaev, "Mixed-symmetry gauge fields in $\operatorname{AdS}(5)$," arXiv:hep-th/0501105; Theor. Math. Phys. 140, 1253 (2004) [Teor. Mat. Fiz. 140, 424 (2004)] arXiv:hep-th/0311212;

[37] Y. M. Zinoviev, "On massive mixed symmetry tensor fields in Minkowski space and (A)dS," arXiv:hep-th/0211233; "First order formalism for mixed symmetry tensor fields," arXiv:hep-th/0304067; "First order formalism for massive mixed symmetry tensor fields in Minkowski and (A)dS spaces," arXiv:hep-th/0306292. 